\title{
From planetesimals to planets: volatile molecules
}

\author{
Ulysse Marboeuf $^{1}$, Amaury Thiabaud $^{1}$, Yann Alibert ${ }^{1,2}$, Nahuel Cabral $^{1}$, and Willy Benz ${ }^{1}$ \\ 1 Physics Institute and Center for Space and Habitability, University of Bern, Bern, Switzerland \\ e-mail: ulysse.marboeuf@space. unibe.ch \\ 2 Observatoire de Besançon, France
}

Received 15 January 2014 / Accepted 20 June 2014

\begin{abstract}
Context. Solar and extrasolar planets are the subject of numerous studies aiming to determine their chemical composition and internal structure. In the case of extrasolar planets, the composition is important as it partly governs their potential habitability. Moreover, observational determination of chemical composition of planetary atmospheres are becoming available, especially for transiting planets. Aims. The present works aims at determining the chemical composition of planets formed in stellar systems of solar chemical composition. The main objective of this work is to provide valuable theoretical data for models of planet formation and evolution, and future interpretation of chemical composition of solar and extrasolar planets.

Methods. We have developed a model that computes the composition of ices in planets in different stellar systems with the use of models of ice and planetary formation.

Results. We provide the chemical composition, ice/rock mass ratio and C:O molar ratio for planets in stellar systems of solar chemical composition. From an initial homogeneous composition of the nebula, we produce a wide variety of planetary chemical compositions as a function of the mass of the disk and distance to the star. The volatile species incorporated in planets are mainly composed of $\mathrm{H}_{2} \mathrm{O}$, $\mathrm{CO}, \mathrm{CO}_{2}, \mathrm{CH}_{3} \mathrm{OH}$, and $\mathrm{NH}_{3}$. Icy or ocean planets have systematically higher values of molecular abundances compared to giant and rocky planets. Gas giant planets are depleted in highly volatile molecules such as $\mathrm{CH}_{4}, \mathrm{CO}$, and $\mathrm{N}_{2}$ compared to icy or ocean planets. The ice/rock mass ratio in icy or ocean and gas giant planets is, respectively, equal at maximum to $1.01 \pm 0.33$ and $0.8 \pm 0.5$, and is different from the usual assumptions made in planet formation models, which suggested this ratio to be $2-3$. The C:O molar ratio in the atmosphere of gas giant planets is depleted by at least $30 \%$ compared to solar value.
\end{abstract}

Key words. planets and satellites: formation - planets and satellites: composition - planets and satellites: general

\section{Introduction}

The determination of the chemical composition of planets has been the subject of numerous studies. The study of the composition of volatile molecules incorporated in planets is an important parameter in planetary internal structure and thermal evolution models of these bodies. Numerous studies focused on the internal structure and chemical composition (mainly atmospheres or ices) of solar (Gautier et al. 2001a,b; Hersant et al. 2004) and extrasolar planets (Hersant et al. 2004; Marboeuf et al. 2008; Bond et al. 2010; Mousis et al. 2010; Johnson et al. 2012). Since the discovery of the first extrasolar planet in 1995, more than 1000 have been discovered by radial velocity measurements, microlensing experiments, and photometric transit observations (Baraffe et al. 2010; Lunine 2011; Griffith et al. 2011). These exoplanets cover a wide range of masses and sizes, among which exoplanets of sub-Mercury sizes (Kepler 37c, Barclay et al. 2013) or a few tens of Jupiter masses (see Udry \& Santos 2007, for a review). Some molecular species such as $\mathrm{H}_{2} \mathrm{O}, \mathrm{CO}$, $\mathrm{CO}_{2}$, and $\mathrm{CH}_{4}$ have already been detected in the troposphere and/or stratosphere of extrasolar giant planets (Beaulieu et al. 2008, 2010; Barman 2007, 2008; Burrows et al. 2005, 2007, 2008; Charbonneau et al. 2002, 2005, 2008; Désert et al. 2009; Fortney \& Marley 2007; Griffith et al. 2011; Grillmair et al. 2008; Lee et al. 2012; Madhusudhan \& Seager 2009, 2010, 2011; Madhusudhan et al. 2011; Madhusudhan 2012; Snellen et al. 2008; Swain et al. 2008, 2009a,b; Tinetti et al. 2007, 2010, 2012; Waldmann et al. 2012). Some numerical studies have also been developed to theoretically predict and explain these observations (e.g., Marboeuf et al. 2008; Johnson et al. 2012) and the implication of this composition on the planetary spectra (Moses et al. 2013) that could be observed from Earth. Moreover, the Exoplanet Characterisation Observatory (EChO), will be the first dedicated mission to investigate the physics and chemistry of exoplanetary atmospheres in order to characterize the physical conditions of their formation and evolution. However, none of these studies has computed the planetary formation in a self-consistent way.

We have recently extended our planet formation model to the formation of planetary systems (see Fortier et al. 2013; Alibert et al. 2013), and our aim, in this paper and a companion one (Thiabaud et al. 2014, hereafter Paper I), is to determine the composition of planet, in a way that is self-consistent with the formation process as modeled in our approach. We have first computed the composition of planetesimals for a population of protoplanetary disk (see Marboeuf et al. 2014, hereafter Paper II), and at any distance to the central star, considering both the icy species (topic of this paper), and refractory species (see Paper I). In this paper, we combine the results of the formation model (which provides the amount of planetesimals accreted by every planet as a function of the distance to the central star) with the composition model presented in the Paper II, to derive the final composition of planets. The main objective of this work is therefore to provide valuable theoretical data for models of planet formation and evolution, and future interpretation of chemical composition of both solar and extrasolar planets.

This article is organized as follows. In a first step (Sect. 2), we present the planetary formation model. In a second step 
(Sect. 3), we present the physical assumption and parameters adopted. We then calculate the chemical composition of planets formed in the protoplanetary disk (Sect. 4), and discuss the results (Sect. 5). The last section (Sect. 6) is devoted to conclusions.

\section{Description of the physical models used in this work}

In order to determine the chemical composition of planets we consider several planetary systems assumed to emerge from a protoplantary disk whose initial density profile, mass, and lifetime is different, and follows, as closely as possible, observational characteristics (see Mordasini et al. 2009; Alibert et al. 2013). We assume, when computing the formation of planets, that planetesimals are already formed at the beginning of the planetary system formation process (initial time of the model) by a process that is presently still a matter of research. Planetesimals are believed to form from the coagulation of small grains (icy and/or rocky), whose chemical composition is itself provided by the condensation sequence of gas in the protoplanetary disks (see Paper II). This provides us with the composition of grains, which is also assumed to be identical to that of planetesimals formed at the same location in the disk. This approach is obviously simplified, since the chemical composition of planetesimals results from the condensation/clathrate sequence at the location of the grains (see Paper II), and we do not include the radial drift of small icy grains. We then use our planetary formation model (Alibert et al. 2005, 2013; Mordasini et al. 2009, 2012a,b; Fortier et al. 2013) to compute the chemical composition of planets formed in the disk.

\subsection{Model of dynamical planet migration and calculation of the chemical composition of planets}

The initial locations of planetary embryos (ten embryos are considered in the models presented in this paper) are selected at random following a uniform distribution in log. The composition of the planetary embryos (whose mass is on the order of lunar mass $-10^{-2} M_{\oplus}$ ) is assumed to be the same as planetesimals condensed at its location (see Paper II). In other words, we assume that the initial planetary embryos are formed locally out of planetesimals formed at the same location.

The model of planet formation allows us to determine the formation path of the ten initial embryos in the protoplanetary disk and their growth during their migration for several disks (different initial surface densities $\Sigma_{0}\left(T_{0}, P_{0}, r\right)^{1}$ of the disk), and for different initial distances to the star. The mass of planetesimals accreted is given by

$\frac{\mathrm{d} M_{p}(r)}{\mathrm{d} t}=\pi R_{\mathrm{c}}^{2} \Sigma_{p} \Omega F_{g} \quad\left(\mathrm{~kg} \mathrm{~s}^{-1}\right)$,

where $\Sigma_{p}\left(\mathrm{~g} \mathrm{~cm}^{-2}\right)$ is the surface density of solids in feeding zone, $R_{\mathrm{c}}(\mathrm{AU})$ the capture radius of the planetesimal, $F_{g}$ the focusing factor, and $\Omega$ the Keplerian frequency

$\Omega^{2}=\frac{G M_{*}}{r^{3}} \quad\left(\mathrm{~s}^{-2}\right)$

with $r$ the distance to the star, $G$ the gravitational constant, and $M_{*}$ the mass of the central star, assumed to be solar in this study.

\footnotetext{
$1 \quad \Sigma_{0}$ is the initial surface density at $5.2 \mathrm{AU}$.
}

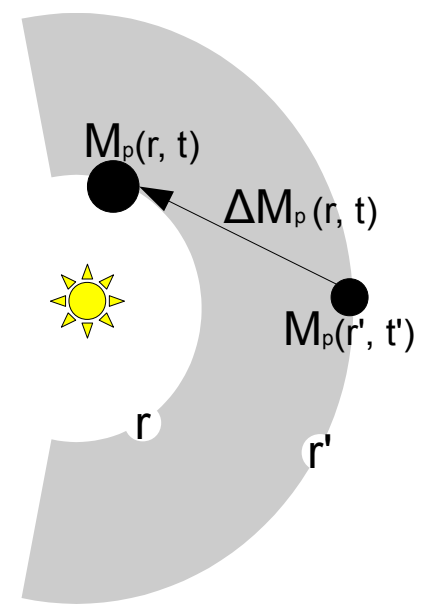

Fig. 1. Schematic view of the migration and grow of a protoplanet by accretion of planetesimals in the protoplanetary disk between positions to the star $r$ and $r^{\prime}$.

During the migration of the planet in the protoplanetary disk, from a distance $r^{\prime}$ to a distance $r$ from the star (see Fig. 1), the variation of the mass $\Delta m_{x}^{p}(r)$ of the species $x$ in the planet $p$ after accretion of planetesimals is defined as

$\Delta m_{x}^{p}(r, t)=\Gamma_{x}^{g}(r) \Delta M_{p}(r, t) \quad(\mathrm{kg})$,

where $\Delta M_{p}(r, t)$ is the mass of planetesimals accreted by the planet $p$ at time $t$ and $\Gamma_{x}^{g}(r)$ the chemical abundance in planetesimals of species $x$.

At each time step, we compute the mass $M_{p}(r, t)$ of the planet $p$ and the mass $m_{x}^{p}(r, t)$ of volatile molecules $x$ in the planet $p$ after accretion of new material $\Delta M_{p}(r, t)$ at the position $r$ and time $t$,

$M_{p}(r, t)=M_{p}\left(r^{\prime}, t^{\prime}\right)+\Delta M_{p}(r, t) \quad(\mathrm{kg})$,

where $M_{p}\left(r^{\prime}\right)$ is the mass of the planet at the distance $r^{\prime}$ and time $t^{\prime}$ from the star before accretion of planetesimals and

$m_{x}^{p}(r, t)=m_{x}^{p}\left(r^{\prime}, t^{\prime}\right)+\Delta m_{x}^{p}(r, t) \quad(\mathrm{kg})$

with $m_{x}^{p}\left(r^{\prime}, t^{\prime}\right)$ the mass of volatile molecules $x$ in the planet $p$ at time $t^{\prime}$ before accretion of planetesimals. At the end of the computation, the molar ratio of volatile molecule $x$ relative to all volatile molecules $\Gamma_{x}^{p}(r)$ is then given by the relation

$\Gamma_{x}^{p}(r)=\frac{m_{x}^{p}(r)}{\sum m_{x}^{p}(r)}$.

The process of accretion of planetesimals onto embryos is assumed to retain the original mass of species initially incorporated in icy bodies. The abundance of volatile molecules initially contained in planetesimals does not undergo chemical changes during the melting, the sublimation, and break up of bodies onto planets. Moreover, the migration of planets toward the star which could induce chemical reactions due to an increase in the surface temperature and temperature-pressure of planetary atmospheres is assumed not to change the abundances of species in planets.

\section{Physical assumptions and parameters adopted in models}

In this paper, we focus on the same volatile components used in the study of planetesimals (see Paper II): $\mathrm{H}_{2} \mathrm{O}, \mathrm{CO}_{2}$, 
Non-irradiated model

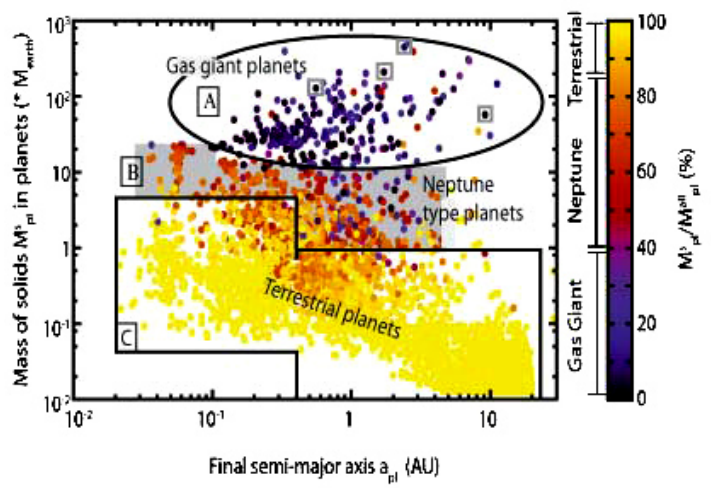

Irradiated model

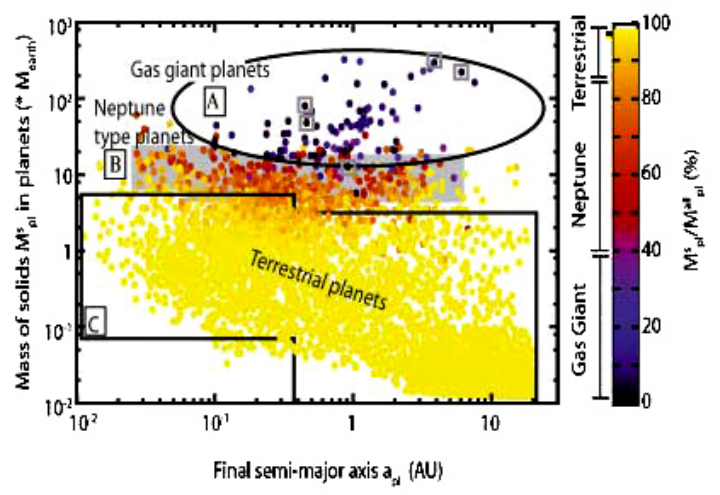

Fig. 2. Mass ratio of solids $M_{p l}^{s}$ (ices, rocks, and refractory organics) relative to the total planetary mass $M_{p l}^{\text {all }}$ as a function of their final semi-major axis $a_{p l}$ and solid mass $M_{p l}^{s}$, for non-irradiated (left panel) and irradiated (right panel) models. The light gray boxes are stars with total masses greater than 13 Jovian mass which could be brown dwarfs.

$\mathrm{CO}, \mathrm{CH}_{3} \mathrm{OH}, \mathrm{CH}_{4}, \mathrm{NH}_{3}, \mathrm{~N}_{2}$, and $\mathrm{H}_{2} \mathrm{~S}$. These volatile components are the major abundant species observed in comets (Bockelée-Morvan et al. 2004; Mumma \& Charnley 2011) and planets. We assume that all volatile molecules are composed of H, O, C, N, and S atoms in solar abundances (Lodders 2003). The chemical composition of planets is determined by taking into account both refractory and volatile components. Refractory elements include both minerals and refractory organics (see Paper I for details). Based on the study on the chemical composition of planetesimals, we study several extremes cases by varying parameters based on the formation of clathrates and refractory organics before the condensation of volatile species at lower temperature, the abundance of $\mathrm{CO}$ (rich $\mathrm{CO}$ and poor $\mathrm{CO}$ models assuming $\mathrm{CO}: \mathrm{CO}_{2}=5: 1$ and $1: 1$, respectively), and the radiation through the disk. As shown in Paper II, the formation of clathrates changes the chemical composition of planetesimals by increasing the abundance of $\mathrm{CO}, \mathrm{CH}_{4}, \mathrm{H}_{2} \mathrm{~S}$, and $\mathrm{N}_{2}$. The formation of refractory organics before the condensation of ices induces a lower amount of ices of several tens of $\%$ in planetesimals compared to models without organics. The abundance of $\mathrm{CO}$ in planetesimals decreases in parallel to that in the disk. The irradiation of the disk (hereafter irradiated models) shifts the ice line positions of all volatile species by about 0.5-1 AU farther in the disk and reduces the amount of highly volatile species incorporated in planetesimals such as $\mathrm{CH}_{4}, \mathrm{CO}$, and $\mathrm{N}_{2}$, compared to non-irradiated models. As discussed in Paper II, the irradiated and non-irradiated models do not properly model the physics in the disk, but allow us to frame the thermodynamic conditions of ice formation in the cooling disk and migration of planets (see Paper I) respectively near and far away from the star. The chemical processes which could change abundances of volatile species in warm surface layers of the protoplanetary disk are not taken into account in this study. All the volatile molecules $\left(\mathrm{H}_{2} \mathrm{O}\right.$, $\mathrm{CO}_{2}, \mathrm{CH}_{3} \mathrm{OH}, \mathrm{CH}_{4}, \mathrm{NH}_{3}$, and $\mathrm{H}_{2} \mathrm{~S}$ ), except $\mathrm{CO}$ and $\mathrm{N}_{2}$, are easily destroyed in the gas phase of the surface layers of the disk (see Visser et al. 2011) by reactions with UV and X-ray photons from the central star as well as UV photons and cosmic rays originating from the interstellar medium. They do not keep high abundances once they are evaporated from grains (Doty et al. 2002; Nomura \& Millar 2004). However, grain surface reactions in the disk will recover the loss, and the abundances of volatile molecules in ice may not be very different from those adopted in planetesimals for this study (see Paper II for more explanation).

Finally, we analyze the chemical composition of planets formed in the different synthesis planetary systems presented in Alibert et al. (2013) using 16 different initial chemical compositions of planetesimals (see Paper II) and using different initial surface densities at 5.2 AU (varying from $3 \mathrm{~g} \mathrm{~cm}^{-2}$ to about $700 \mathrm{~g} \mathrm{~cm}^{-2}$ ), density slopes (varying from 0.4 to 1.1 ), and characteristic scaling radius (varying from 14 AU to $198 \mathrm{AU}$ ) of the disk (see Sect. 3.3 in Paper I, and Alibert et al. 2013). The different initial physical conditions of disks induce different initial thermodynamic conditions of ice formation (see Paper II) and planetary evolution as shown in Fig. 7 of Paper II. As mentioned in Sect. 2, each simulation starts with ten planetary embryos that grow and interact within the same protoplanetary disk. The characteristics of the protoplanetary disk (namely mass, lifetime, and gas-to-solids ratio) are varied from one simulation to the other in order to explore the different possible initial conditions. Depending on the characteristics of the simulation, the composition of planetesimal will change, as well as the parts of the disk sampled by the different planets, providing a diversity of composition.

At the beginning of the simulations, the protoplanets have a lunar mass (mass of about $10^{-2} M_{\oplus}$ ) and the chemical composition of the planetesimals formed at that location. With time, protoplanets migrate and grow (see Sect. 2.1) by accretion of planetesimals whose chemical composition is described in Paper II. As a result, they are enriched or depleted in volatile molecules compared to initial protoplanets. The mass of planetesimals accreted by a planet depends on the solid surface density and the protoplanet's efficiency in capturing solid planetesimals. Unless stated explicitly, figures described in Sect. 4 present the mass of solid components (ices, rocks, and refractory organics) $M_{p l}^{s}$ in planets and do not include the mass of the gas. This means that we do not include in our model the possibility of capture of volatile species as part as the accreted gas (which is therefore assumed to contain only $\mathrm{H}$ and $\mathrm{He}$ ).

\section{Chemical composition of planets}

\subsection{Type of planets and Ice/rock mass ratio}

Mass fraction of the solid component of planets. Figure 2 shows the mass ratio of solid components (core of the planet) $M_{p l}^{s}$ relative to the total mass of the planet $\left(M_{p l}^{\text {all }}\right.$, including solid components and envelope) in planets as a function of their final semi-major axis $a_{p l}$ and of the mass of the solid 
Non-irradiated model
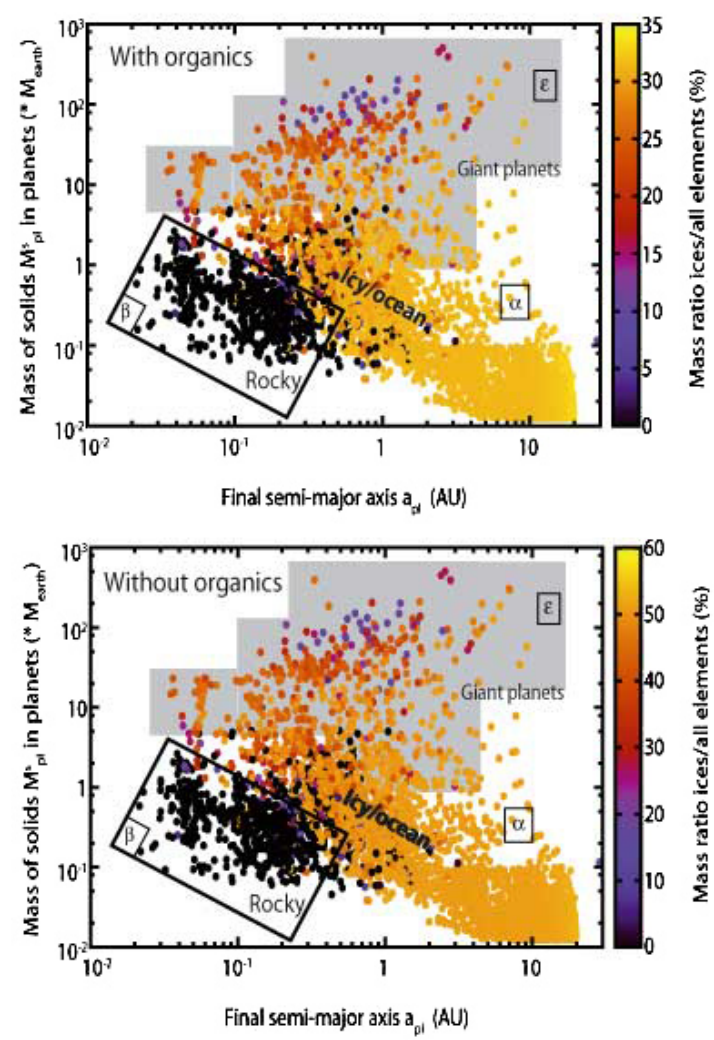

Irradiated model
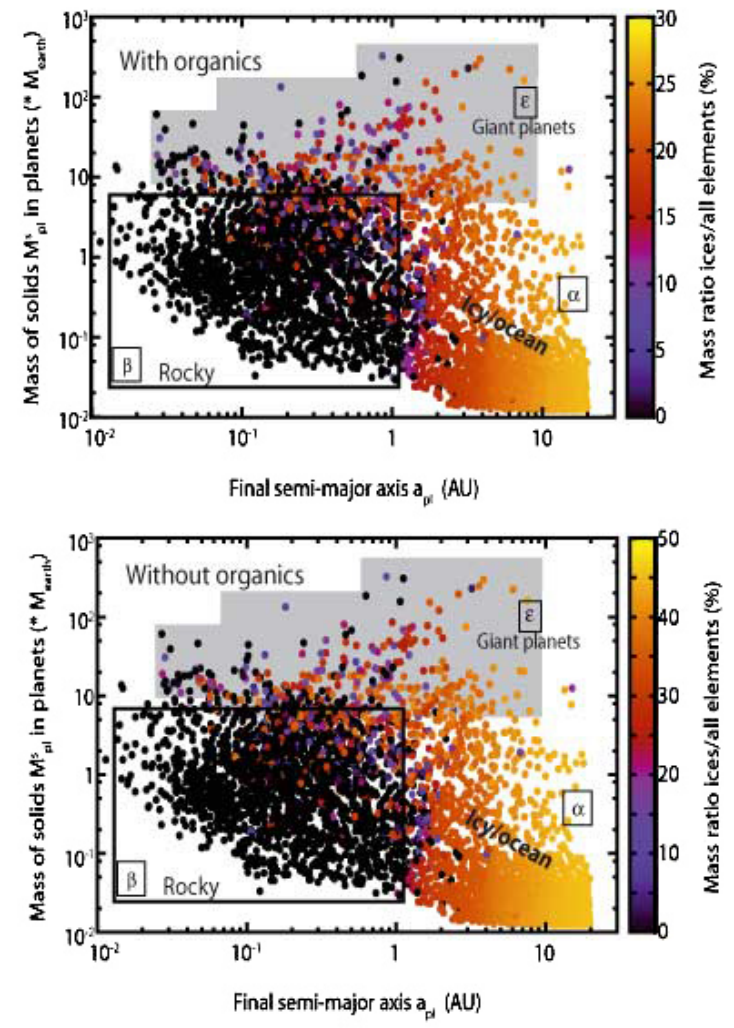

Fig. 3. Average mass ratio of volatile molecules relative to the total solid mass of planets as a function of the final semi-major axis $a_{p l}$ and the mass of the solid component $M_{p l}^{s}$, for non-irradiated (left panel) and irradiated (right panel) models, with (upper panel) and without (lower panel) refractory organics.

components $M_{p l}^{s}$, for non-irradiated (left panel) and irradiated (right panel) models. For both models, the population of planets are similar and can be divided into three groups. The first (hereafter group $A$ ) corresponds to gaseous giant planets with $M_{p l}^{s} \geq 20 M_{\oplus}$ for both non-irradiated and irradiated models. The mass of the gas envelope is at least $60 \mathrm{wt} \%$ of the total mass $M_{p l}^{\text {all }}$ of planets (black and blue dots). The second group (gray area, hereafter group $B$ ) includes less massive planets, with a mass in the range of $\approx 1-5 M_{\oplus} \leq M_{p l}^{s} \leq 10-20 M_{\oplus}$ (resp. $\approx 5 M_{\oplus} \leq M_{p l}^{s} \leq \approx 20 M_{\oplus}$ ) for non-irradiated (resp. irradiated) models. The mass of solids $M_{p l}^{s}$ included in these planets varies from about $40 \mathrm{wt} \%$ to about $90 \mathrm{wt} \%$ of the total mass $M_{p l}^{\text {all }}$ of planets (purple and orange dots). The last group (hereafter group $C$ ) includes low mass planets, with $M_{p l}^{s} \leq 1-5 M_{\oplus}$ (resp. $M_{p l}^{s} \leq 5-10 M_{\oplus}$ ) for non-irradiated (resp. irradiated) models and a very small mass fraction of gas $(\leq 10 \%$, yellow dots). Some planets (light gray boxes, 4 in non-irradiated and 5 in irradiated models) have total masses greater than 13 Jovian mass and could be brown dwarfs rather than gas giant planets. As can be seen in Fig. 2, the mass of gas increases with the mass of heavy elements, a result typical of planets formed in the coreaccretion model. The major change with the irradiated models is the number of low and high mass planets. The number of low/high mass planets increases/decreases slightly compared to

\footnotetext{
2 The term "solid component" does not necessarily mean that these elements remain in the solid phase (the planetary core), but only that they have been captured under the form of planetesimals. Similarly, the term "mass of gas" refers to the mass that has been accreted under the form of gas, including possible small dust.
}

the non-irradiated models. As shown below (see Fig. 3), this is due to the depletion of ices in the disk and to the change of the migration pathway of planets between non-irradiated and irradiated models (see Fig. 4).

We note that the maximum mass of heavy elements in gaseous giant planets, which reaches about $500 M_{\text {earth }}$, is probably favored by the hypothesis that all planetesimals have the same radius (on the order of $100 \mathrm{~m}$ ) and this radius does not evolve with time (see Fortier et al. 2013; Alibert et al. 2013). A size distribution of planetesimals and a radius evolution would probably change the maximum mass of planets formed.

Fraction of ices relative to the total solid mass. Figure 3 shows the average mass ratio of volatile molecules, relative to the total mass of heavy elements (volatile molecules, and rocks (including minerals and organics compounds), excluding the gaseous envelope) as a function of the mass of solid components $M_{p l}^{s}$ and the semi-major axis $a_{p l}$ of planets at the end of the computation, for non-irradiated and irradiated models, both with and without refractory organics, and taking into account all chemical variations ( $\mathrm{CO}: \mathrm{CO}_{2}$ variation and clathrate formation). Whatever the model used, the planets are chemically divided into three parts and cover approximately the subdivisions $A-C$ described in Fig. 2:

1. The first area (hereafter area $\alpha$ ) is composed of planets with a solid mass $M_{p l}^{s}$ ranging from $0.01 M_{\oplus}$ to $5 M_{\oplus}$ (resp. 0.01 $M_{\oplus}$ to about $10 M_{\oplus}$ ) for non-irradiated (resp. irradiated) models. These planets are mainly icy or ocean planets (hereafter icy/ocean planets) with low mass and a very small gaseous envelope (see Fig. 2). They are characterized by a 

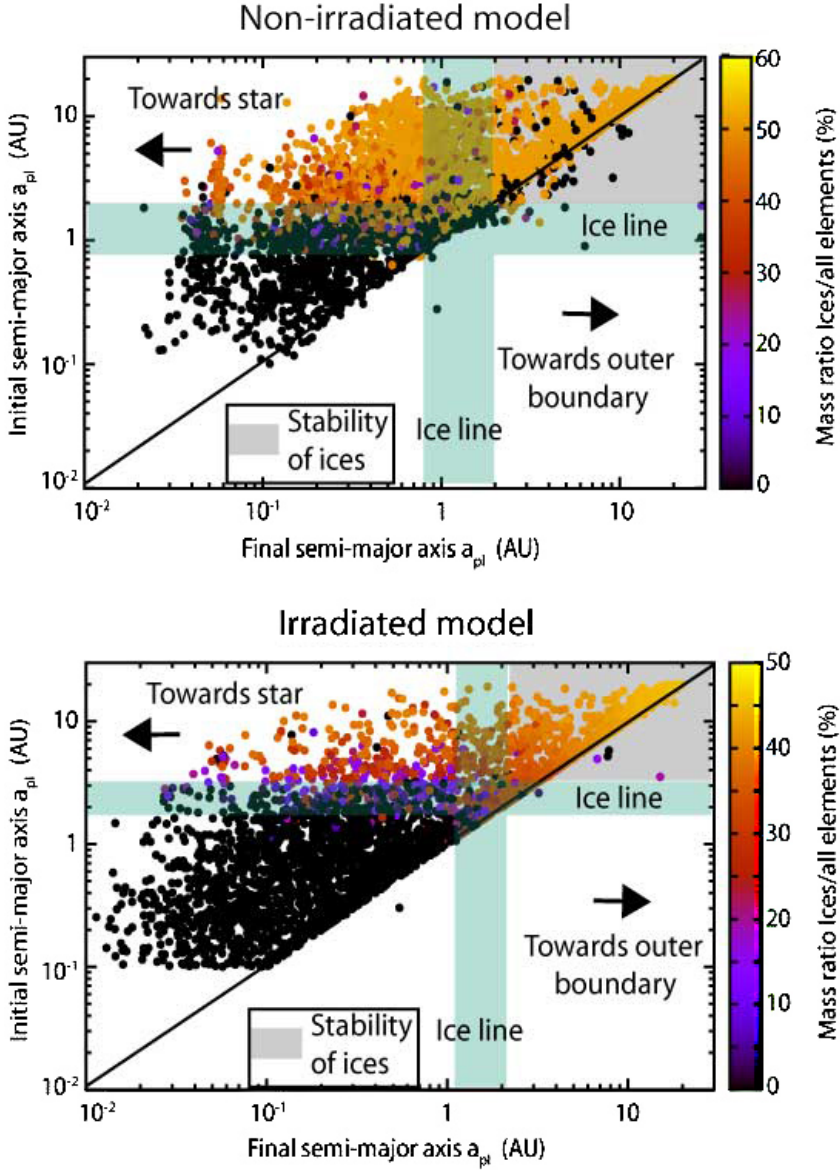

Fig. 4. Mass ratio of volatile molecules relative to the mass of solids in the planets, as a function of initial and final semi-major axis $a_{p l}$, for nonirradiated (upper panel) and irradiated (lower panel) models. The blue area corresponds to the ice water line position for most simulations. Gray area corresponds to the zone of stability of ices beyond the ice line. Models without refractory organics assume $\mathrm{CO}: \mathrm{CO}_{2}=1: 1$.

final semi-major axis $a_{p l}$ ranging from $0.4 \mathrm{AU}$ to $20 \mathrm{AU}$ (resp. $1 \mathrm{AU}$ to $20 \mathrm{AU}$ ) for non-irradiated (resp. irradiated) models. We note that some icy/ocean planets are outside of the ranges described; some planets are located near the star between $0.04 \mathrm{AU}$ and $0.4 \mathrm{AU}(0.1 \mathrm{AU}$ and $1 \mathrm{AU})$ for nonirradiated (resp. irradiated) models and could be ocean planets. Typically, the solid component of icy/ocean planets is composed of $31 \pm 8 \mathrm{wt} \%$ (resp. $50 \pm 12 \mathrm{wt} \%$ ) of ices in non-irradiated models with (resp. without) refractory organics. Variations represent the standard deviation $2 \sigma$ of values, taking into account all chemical changes (formation of clathrates, $\mathrm{CO}: \mathrm{CO}_{2}$ variation). Planets in irradiated models (without refractory organics compounds) present lower ratios with a radial gradient of the amount of ices. Average values $( \pm 2 \sigma)$ of the fraction of ices in planets are $23 \pm 10 \mathrm{wt} \%$ (resp. $40 \pm 14 \mathrm{wt} \%$ ) in icy/ocean planets, with (resp. without) refractory organics. This leads to icy/ocean planets with average ice/rock mass ratio varying from $0.30 \pm 0.16$ (irradiated models with refractory organics) to $1.01 \pm 0.33$ (nonirradiated models without refractory organics). Such values frame the ones of some icy satellites and KBOs in the solar system: densities of Jovian icy satellites Ganymede and Callisto; Saturn's moon Titan; Neptune's moon Triton; and KBOs Quaoar, Orcus, Eris, and the Pluto-Charon binary $\left(\approx 1.8-2.3 \mathrm{~g} \mathrm{~cm}^{-3}\right)$ suggest comparable masses of rock and ices (Showman \& Malhotra 1999; Buie et al. 2006; Person et al. 2006; Brown \& Schaller 2007; McKinnon et al. 2008; Sotin et al. 2009; Lunine et al. 2010).

2. The second area (hereafter area $\beta$ ) contains dry terrestrial planets (little or no $\mathrm{H}_{2}$ and $\mathrm{He}$ gas) with at maximum 1-2 wt\% of volatile molecules (mainly $\mathrm{H}_{2} \mathrm{O}$ ) for all models. This area is largely composed of rocky planets that represent approximately $20 \%$ (resp. $\approx 40 \%$ ) of all the planets for non-irradiated (resp. irradiated) models. The solid mass $M_{p l}^{s}$ and positions $a_{p l}$ of these planets vary from $0.5 M_{\oplus}$ to about $2 M_{\oplus}$ (resp. in the range $0.3-10 M_{\oplus}$ ), and from 0.02 AU to 0.5 AU (resp. from 0.01 AU to 1.5 AU), for non-irradiated models (resp. irradiated models). We note that there are some rocky planets outside of this area with masses varying from $0.5 M_{\oplus}$ to $5 M_{\oplus}$ (resp. $15 M_{\oplus}$ ) and positions varying from $0.05 \mathrm{AU}$ to $3 \mathrm{AU}$ (resp. $3 \mathrm{AU}$ ) for nonirradiated (resp. irradiated) models.

3. The third area (hereafter area $\epsilon$ ) is composed of gas giant and Neptune-type planets (hereafter called giant planets) with a solid mass $M_{p l}^{s}$ ranging from $1-5 M_{\oplus}$ to $500 M_{\oplus}$ and a semimajor axis $a_{p l}$ varying from $0.03 \mathrm{AU}$ to $10 \mathrm{AU}$, for both irradiated and non-irradiated models. The average mass fraction of volatile molecules (relative to the total solid mass) for planets in this area is $26 \pm 11 \mathrm{wt} \%$ (resp. $43 \pm 17 \mathrm{wt} \%$ ) for non-irradiated models with (resp. without) refractory organics. For irradiated models, this fraction decreases and represents about $16 \pm 13 \mathrm{wt} \%$ (resp. $28 \pm 23 \mathrm{wt} \%$ ) with (resp. without) refractory organics. This leads to planets with average ice/rock mass ratios varying from $0.19 \pm 0.2$ (irradiated models with refractory organics) to $0.8 \pm 0.46$ (nonirradiated models without refractory organics).

In summary, we obtain for both irradiated and non-irradiated models, three types of planets: giant planets $\left(1-5 \leq M_{p l}^{s} \leq\right.$ $\left.500 M_{\oplus}\right)$, rocky planets $\left(M_{p l}^{s} \leq 2-5 M_{\oplus}\right.$, and $\left.a_{p l} \leq 0.01-2 \mathrm{AU}\right)$, and icy/ocean planets $\left(M_{p l}^{s} \leq 5-10 M_{\oplus}\right.$, and $\left.a_{p l} \geq 0.04 \mathrm{AU}\right)$. The mass, position relative to the star, and the amount of ices in planets are mainly functions of the surface density of the disk, the formation of refractory organics, and the radiation in the disk. The full irradiation of the disk shifts the ice line positions (see Paper II) and decreases the amount of ices incorporated in planets by up to $5-15 \mathrm{wt} \%$ compared to non-irradiated dics. Moreover, the change of the thermodynamic conditions in the disk for irradiated models changes the pathway of planets which migrate more to the star (see Fig. 4 which shows the mass ratio of ices relative to the total solid mass of planets, models without organics, for the non-irradiated and irradiated models as a function of their initial and final semi-major axis $a_{p l}$ ) and incorporate more rocks than ices. This results in a greater number of less massive and rocky planets near the star and fewer gas giant planets in the disks. We also note that whatever the models, planets migrate mainly towards the star while only a few migrate outwards.

Figures 5 and 6 present the average mass ratio of ices, minerals, and refractory organics relative to all solid elements, and the ice/rock mass ratio (both with standard deviation $\pm 2 \sigma$ ) in icy/ocean and giant planets, respectively, and for all models, taking into account the formation of clathrates and the variation of the abundance of $\mathrm{CO}$ in planetesimals.

\subsection{Abundances of volatile molecules and atoms in planets}

Composition of ices (molar fraction of species relative to all ices). The chemical composition of species in planets is 

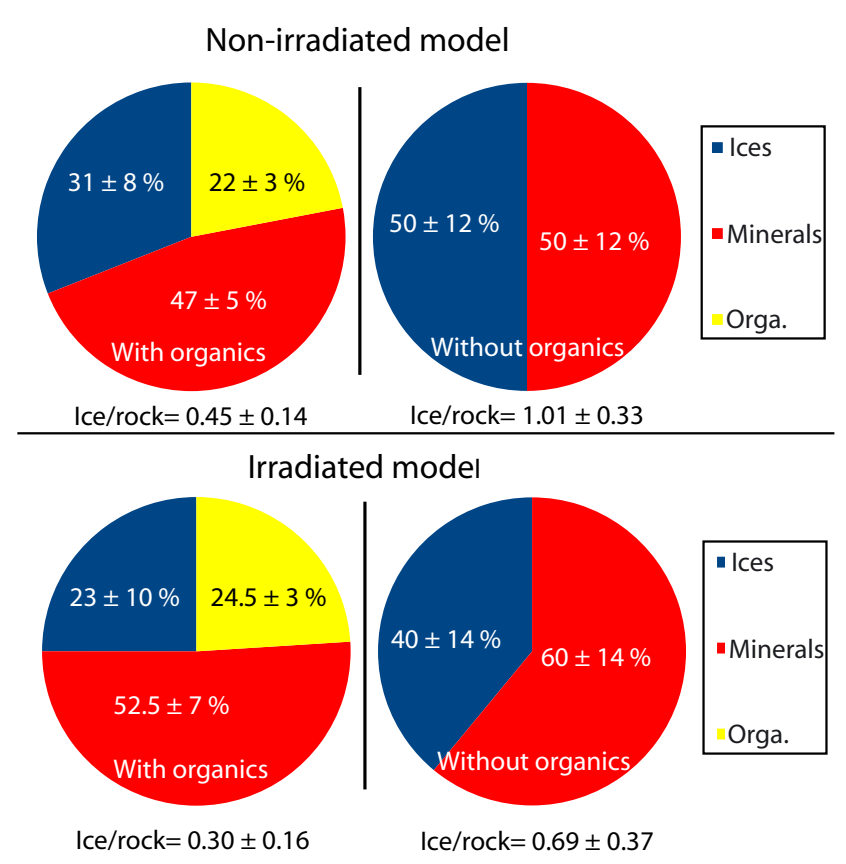

Fig. 5. Average (with standard deviation $2 \sigma$ ) mass ratio of ices, minerals, and refractory organics in icy/ocean planets for all models, taking into account chemical variations $\left(\mathrm{CO}: \mathrm{CO}_{2}\right.$ variation and clathrate formation).

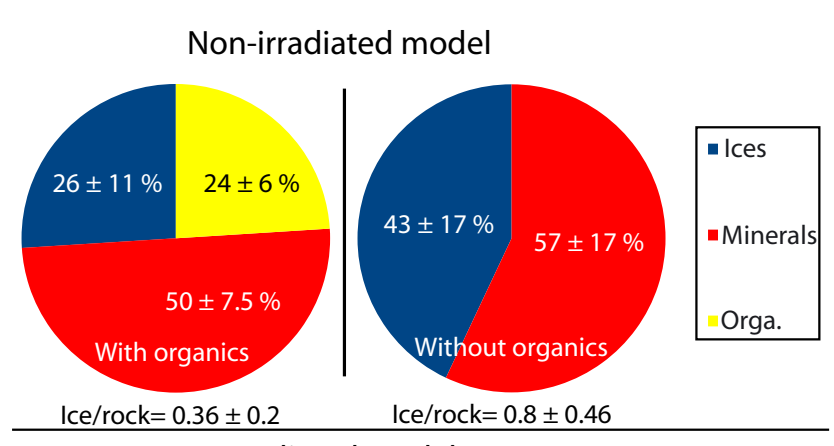

Irradiated model
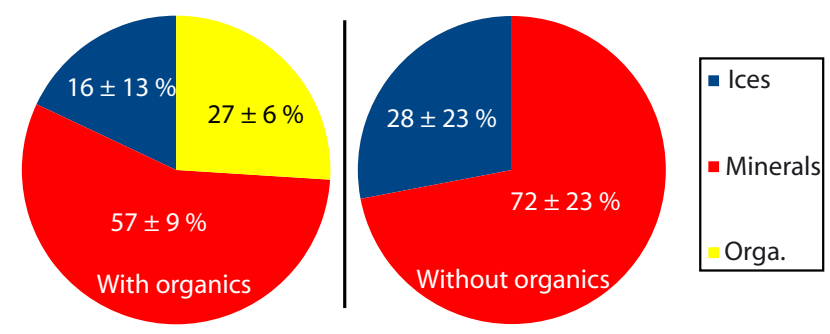

Ice/rock $=0.195 \pm 0.18$

respectively, the average molar ratio of species $\mathrm{H}_{2} \mathrm{O}, \mathrm{CH}_{3} \mathrm{OH}$, $\mathrm{NH}_{3}, \mathrm{CO}_{2}, \mathrm{H}_{2} \mathrm{~S}, \mathrm{CH}_{4}, \mathrm{CO}$, and $\mathrm{N}_{2}$ in planets as a function of final semi-major axis $a_{p l}$ and the mass of the solid component $M_{p l}^{s}$, for irradiated (right panel) or non-irradiated (left panel) models, taking into account all the chemical changes $\left(\mathrm{CO}: \mathrm{CO}_{2}\right.$ variation, clathrate formation). Figure 9 compiles all the results by showing the average molar ratio $( \pm 2 \sigma)$ of species $X$ relative to all volatile species in icy/ocean and giant planets, and for all models taking into account the chemical variation (formation of clathrates, $\mathrm{CO}: \mathrm{CO}_{2}$ variation).

Rocky planets (in area $\beta$ ) contain only a few ices that are mainly composed of $\mathrm{H}_{2} \mathrm{O}$ molecules (see Fig. 7), both for irradiated and non-irradiated models. So, the chemical composition of these planets is not discussed further. Planets that contain a mix of different ices are only found in the areas $\alpha$ and $\epsilon$. In these areas, the average molar ratio of $\mathrm{H}_{2} \mathrm{O}$ (the most abundant volatile molecule) relative to all ices is equal at $63 \pm 14 \%$ in icy/ocean planets and increase by about 5\% in giant planets of non-irradiated models (see Fig. 9, area $\epsilon$ ). The standard deviation $2 \sigma$ takes into account the values of all non-irradiated models. Planets in irradiated models contain larger abundances (relative to all species) of $\mathrm{H}_{2} \mathrm{O}$ with average values of $80 \pm 14 \%$ for icy/ocean planets, and $89 \pm 12 \%$ for giant planets. This means that abundances of species more volatile than $\mathrm{H}_{2} \mathrm{O}$ are depleted (relative to $\mathrm{H}_{2} \mathrm{O}$ ) in planets of irradiated models. As explained in Paper II, the irradiated models always contain larger abundances of $\mathrm{H}_{2} \mathrm{O}$ relative to other species, but with continuous radial variations due to irradiation. This is due to a higher temperature in the disk which decreases the amount of highly volatile species incorporated in planetesimals as shown by Figs. 3 and 7 in Paper II. Note, however, that the total amount of $\mathrm{H}_{2} \mathrm{O}$ (and other volatile species) incorporated in irradiated planets decreases compared to the one in non-irradiated models. Remember that the amount of ices incorporated in planets decreases in irradiated compared to non-irradiated models (see Figs. 5 and 6, and Figs. 8 and 11 for planetesimals in Paper II). We also note that the relative (to all volatile species) abundance of $\mathrm{H}_{2} \mathrm{O}$ in irradiated models shows radial variations due to the slow decrease of the temperature of the disk and the corresponding slow increase in relative (to all volatile species) abundances of all the species with the distance to the star (see Figs. 3, 7, and 8 in Paper II).

The molar fractions of the volatile species $\mathrm{CO}_{2}, \mathrm{CH}_{3} \mathrm{OH}$, $\mathrm{NH}_{3}$, and $\mathrm{H}_{2} \mathrm{~S}$ show relative variations of about $10 \%$ (relative to their respecting average abundance) in icy/ocean planets of nonirradiated models (see Figs. 7 and 8). Their average abundance $( \pm 2 \sigma)$ are roughly equal to $8-9 \pm 2 \%$ for both $\mathrm{CO}_{2}$ and $\mathrm{CH}_{3} \mathrm{OH}$, and $3.2 \pm 1 \%$ and $\approx 1 \pm 0.2 \%$, respectively, for $\mathrm{NH}_{3}$ and $\mathrm{H}_{2} \mathrm{~S}$. In irradiated models, the average abundance of these species is equal to or lower by a few percentage points compared to nonirradiated models, but with higher standard deviation. The average abundance of the species $\mathrm{CO}_{2}, \mathrm{CH}_{3} \mathrm{OH}, \mathrm{NH}_{3}$, and $\mathrm{H}_{2} \mathrm{~S}$ in icy/ocean planets are roughly equal to $5.5 \pm 7 \%$ for $\mathrm{CO}_{2}, 10 \pm 3 \%$ for $\mathrm{CH}_{3} \mathrm{OH}$, and $2.6 \pm 2 \%$ and $\approx 0.5 \pm 0.7 \%$ for, respectively, $\mathrm{NH}_{3}$ and $\mathrm{H}_{2} \mathrm{~S}$. In the giant planets, the abundances of the less volatile species shows larger depletion.

In all models, giant planets are depleted in highly volatile species such as $\mathrm{CH}_{4}, \mathrm{CO}$, and $\mathrm{N}_{2}$ compared to icy/ocean planets located far from the star $(\geq 10 \mathrm{AU})$. While the abundance of these species decrease by a few percentage points in giant planets of non-irradiated models, giant planets in irradiated disks are fully depleted (relative abundances inferior to $1 \%$ ) in high volatile species such as $\mathrm{CH}_{4}, \mathrm{CO}$, and $\mathrm{N}_{2}$ (see Figs. 8 and 9). In icy/ocean planets (non-irradiated models), the average abundance of volatile molecules is equal to $1.7 \pm 1 \%$ and $1.2 \pm 1 \%$ 


\section{Non-irradiated model}
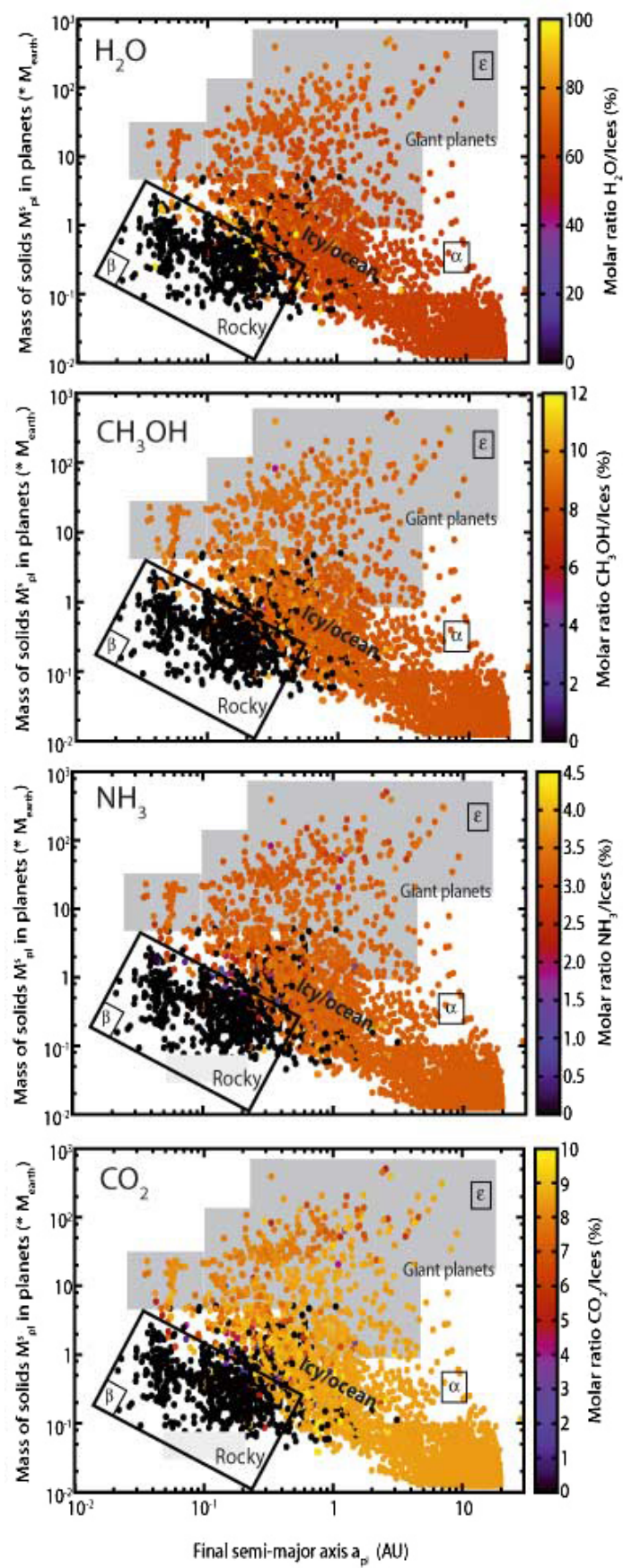

Irradiated model
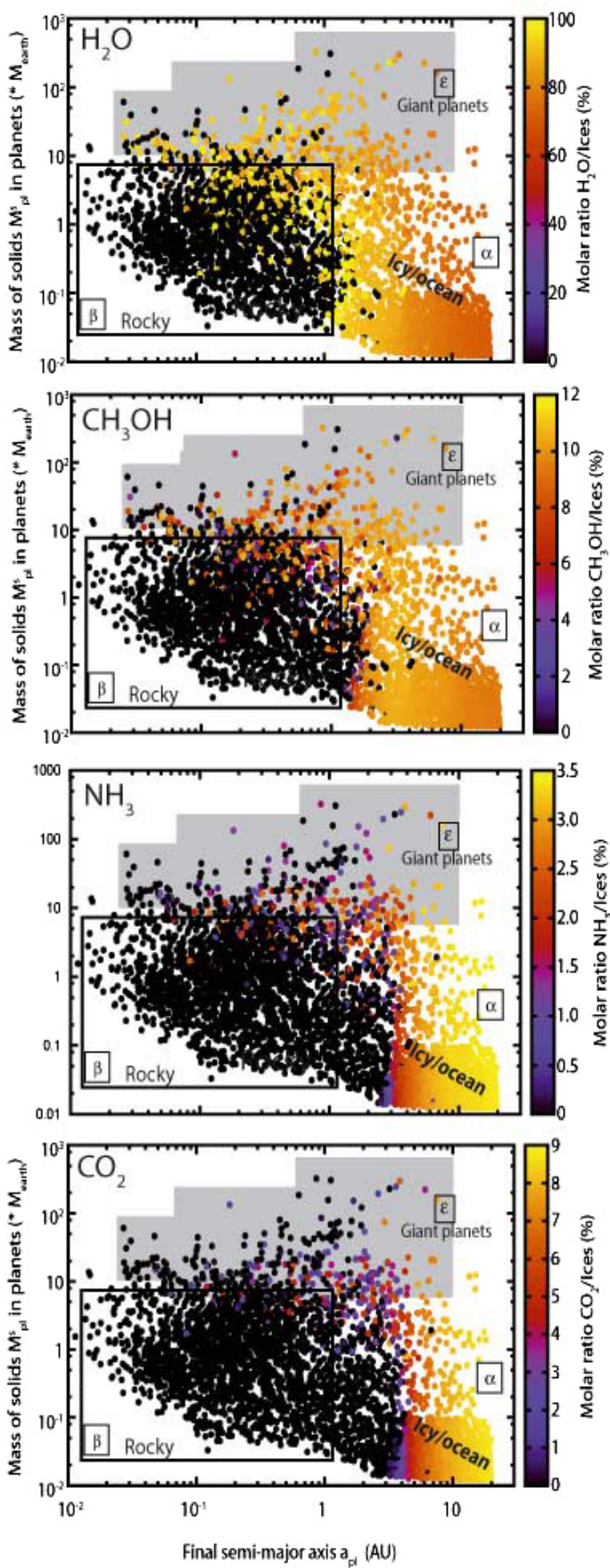

Fig. 7. Average molar ratio of species $\mathrm{H}_{2} \mathrm{O}, \mathrm{CH}_{3} \mathrm{OH}, \mathrm{NH}_{3}$, and $\mathrm{CO}_{2}$ relative to all volatile species in planets as a function of the solid mass $M_{p l}^{s}$ and final semi-major axis $a_{p l}$ for non-irradiated and irradiated models, taking into account all chemical changes $\left(\mathrm{CO}: \mathrm{CO}_{2}\right.$ variation and clathrate formation).

for $\mathrm{CH}_{4}$ and $\mathrm{N}_{2}$, respectively. The $\mathrm{CO}$ abundance in planets (see Fig. 8) vary with the $\mathrm{CO}$ abundance assumed in the solar nebula before the condensation/trapping of species (see Paper II). Taking into account all non-irradiated models, its average value is $13 \pm 16 \%$ in icy/ocean planets. In giant planets, $\mathrm{CO}$ decreases to about $9 \pm 13 \%$. In irradiated models (see Fig. 8), planets present a lower abundance of highly volatile species with a higher standard deviation (see Fig. 9).

Figure 10 synthesizes all the results: it presents the minimum and maximum molar ratio of species $X$ (except $\mathrm{H}_{2} \mathrm{O}$ ) relative to all ices in planets for all models. Table 1 summarizes the average molar fractions of species (relative to all species) for all models.

Icy/ocean planets have approximately the same (homogeneous) chemical composition as planetesimals (see Paper II), because they grow approximately at the same position as the formation of embryos (see Fig. 4), while giant planets have moved in the protoplanetary disk and accreted planetesimals everywhere in the disk. Giant planets have molecular abundances that can vary widely depending on the distance of formation of planets (inside or outside the water ice line) and their migration 


\section{Non-irradiated model}
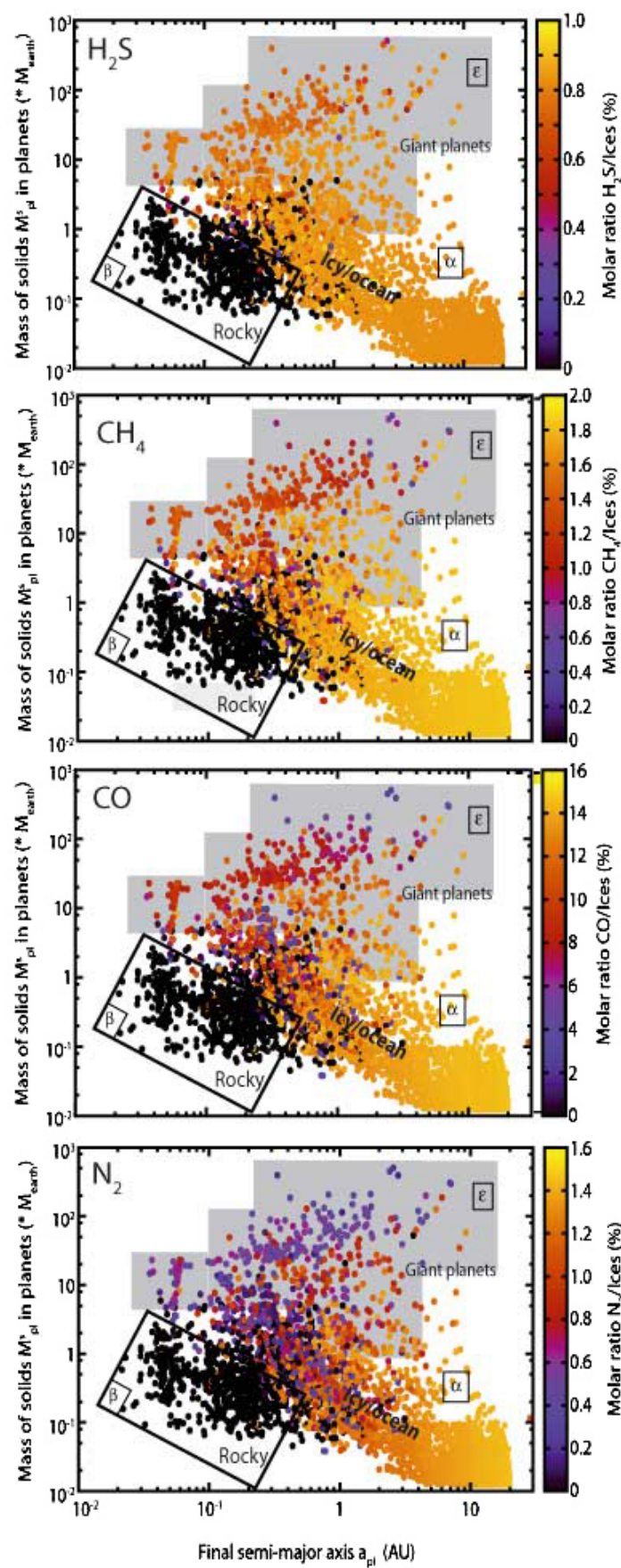

Fig. 8. Same as Fig. 7 but for species $\mathrm{H}_{2} \mathrm{~S}, \mathrm{CH}_{4}, \mathrm{CO}$, and $\mathrm{N}_{2}$.

path in the protoplanetary disk. If these planets are mainly composed of $\mathrm{H}_{2} \mathrm{O}, \mathrm{CO}_{2}, \mathrm{CH}_{3} \mathrm{OH}$, and $\mathrm{CO}$, they show high variations of all abundance species within this region, depending of the pathway of planets in the disk. Compared to the icy/ocean planets, a lot of giant planets are depleted in highly volatile molecules such as $\mathrm{CH}_{4}, \mathrm{~N}_{2}$, and $\mathrm{CO}$ (see Figs. 8 and 9, and Table 1 for synthesis). From icy/ocean planets to giant planets, the abundance of $\mathrm{CO}$ and $\mathrm{CH}_{4}$ is reduced by approximately $20-30 \%$ in non-irradiated models. The $\mathrm{H}_{2} \mathrm{O}$ abundance increases by about $5 \mathrm{wt} \%$. However, some of the giant planets are characterized by abundances of species similar to those of icy/ocean planets, such as $\mathrm{NH}_{3}, \mathrm{CH}_{3} \mathrm{OH}$, and $\mathrm{CO}_{2}$.

\section{Irradiated model}
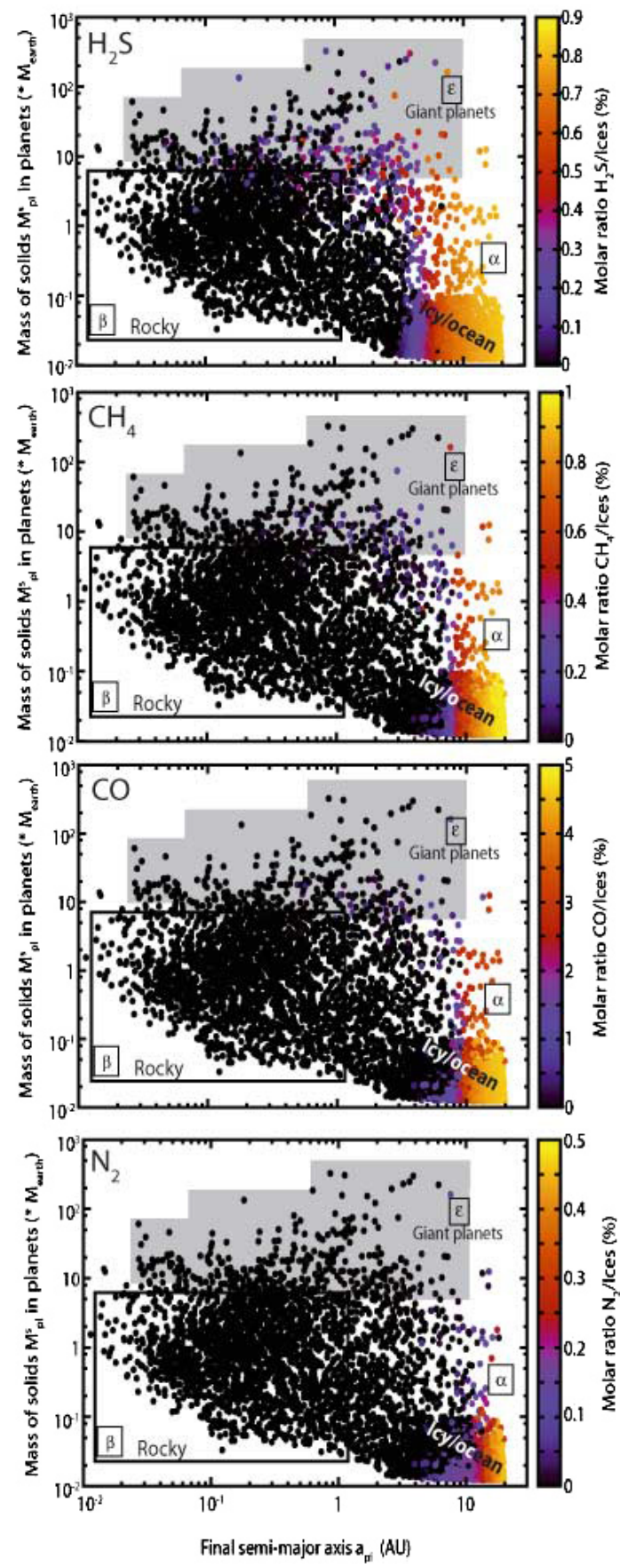

Abundances of the $\mathrm{O}, \mathrm{C}, \mathrm{N}$, and $\mathrm{S}$ atoms relative to $\mathrm{H}_{2}$. In order to compare observational chemical compositions of giant planets and exoplanets to our results, we provide the abundance of atoms $\mathrm{O}, \mathrm{C}, \mathrm{N}$, and $\mathrm{S}$ (initially included in volatile species $\mathrm{H}_{2} \mathrm{O}, \mathrm{CO}, \mathrm{CO}_{2}, \mathrm{CH}_{3} \mathrm{OH}, \mathrm{CH}_{4}, \mathrm{NH}_{3}, \mathrm{~N}_{2}$, and $\mathrm{H}_{2} \mathrm{~S}$ ) relative to $\mathrm{H}_{2}$ in the atmospheres of planets (see Fig. 11), assuming that all volatile species are in the gaseous state of the atmosphere of planets, whatever the position and surface temperatures of planets in the disk. We assume that ices initially incorporated in planetesimals are delivered as ices onto planets, but sublimate fully at the surface of planets after or during their accretion. Figure 11 presents the molar ratio of atoms $\mathrm{O}, \mathrm{C}, \mathrm{N}$, and $\mathrm{S}$, 
Non-irradiated model

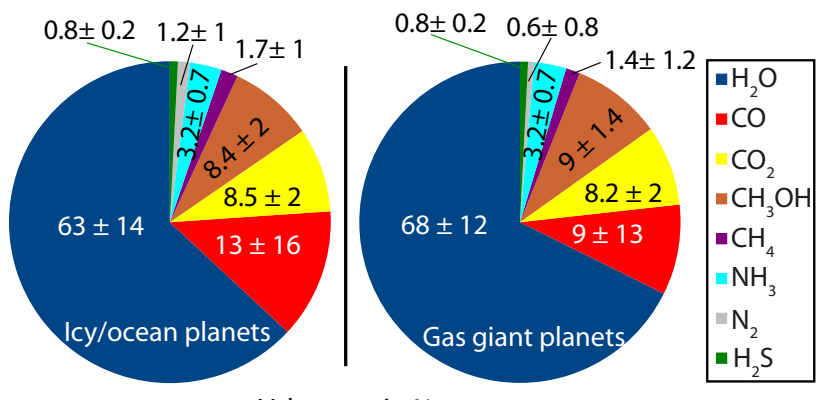

Values are in \%

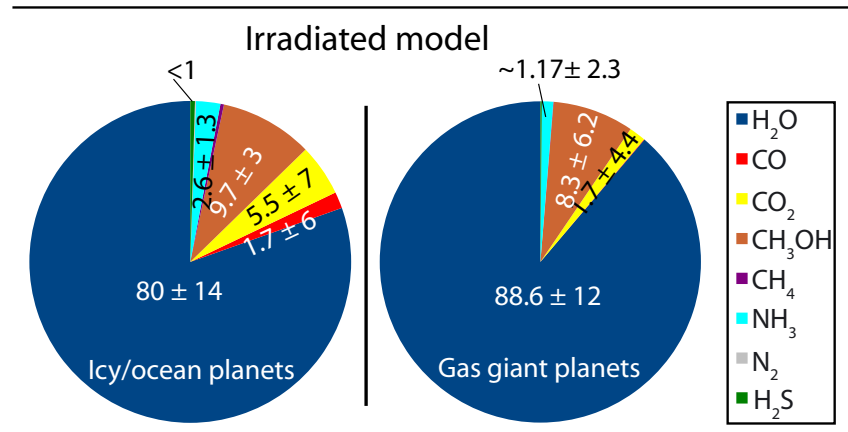

Values are in \%

Fig. 9. Average molar ratio (with standard deviation $2 \sigma$ ) of species $X$ relative to all species in icy/ocean and giant planets for all models, taking into account all chemical changes $\left(\mathrm{CO}: \mathrm{CO}_{2}\right.$ variation and clathrate formation).

Table 1. Average molar ratio of species $X$ (with standard deviation $2 \sigma$ ) relative to all species in planets.

\begin{tabular}{l|cc|cc}
\hline \hline \multirow{2}{*}{$\begin{array}{l}\text { Models } \\
\text { planets }\end{array}$} & \multicolumn{2}{|c|}{ Non-irradiated } & \multicolumn{2}{c}{ Irradiated } \\
Icy/Ocean & Giant & Icy/Ocean & Giant \\
\hline $\mathrm{X}_{p l} /$ Ices & $(\%)$ \\
$\mathrm{H}_{2} \mathrm{O}$ & $63 \pm 14$ & $68 \pm 12$ & $80 \pm 14$ & $88.6 \pm 12$ \\
$\mathrm{CO}$ & $13 \pm 16$ & $9 \pm 13$ & $1.7 \pm 6$ & $0.07 \pm 1$ \\
$\mathrm{CO}_{2}$ & $8.5 \pm 2$ & $8.2 \pm 2$ & $5.5 \pm 7$ & $1.7 \pm 4.4$ \\
$\mathrm{CH}_{4}$ & $1.7 \pm 1$ & $1.4 \pm 1.2$ & $0.37 \pm 1.2$ & $0.03 \pm 0.3$ \\
$\mathrm{H}_{2} \mathrm{~S}$ & \multicolumn{2}{|c|}{$0.8 \pm 0.2$} & $0.48 \pm 0.7$ & $0.14 \pm 0.4$ \\
$\mathrm{~N}_{2}$ & $1.2 \pm 1$ & $0.6 \pm 0.8$ & $0.1 \pm 0.4$ & 0 \\
$\mathrm{NH}_{3}$ & \multicolumn{2}{|c|}{$3.2 \pm 0.7$} & $2.6 \pm 2.3$ & $1.17 \pm 2.3$ \\
$\mathrm{CH}_{3} \mathrm{OH}$ & $8.4 \pm 2$ & $9 \pm 1.4$ & $9.7 \pm 3$ & $8.3 \pm 6.2$ \\
\hline
\end{tabular}

Notes. ${ }^{(*)}$ Ices $=\mathrm{H}_{2} \mathrm{O}+\mathrm{CO}+\mathrm{CO}_{2}+\mathrm{CH}_{4}+\mathrm{H}_{2} \mathrm{~S}+\mathrm{N}_{2}+\mathrm{NH}_{3}+$ $\mathrm{CH}_{3} \mathrm{OH}$.

relative to the total amount of $\mathrm{H}_{2}$ in planets as a function of the total planetary mass $M_{p l}^{\text {all }}$ and the $M_{p l}^{s}: M_{p l}^{\text {all }}$ mass ratio in planets, for all models. Gray dots labeled $J$ and $S$ represent the abundances of atoms C, N, and S for planets Jupiter and Saturn, respectively (Wong et al. 2004; Fletcher et al. 2009). For all atoms, the $\mathrm{X}: \mathrm{H}_{2}$ molar ratio in the atmosphere of planets is almost always greater than that in the molecular cloud that gave birth to the planetary system (ISM value), except for some planets where it can become inferior. For giant planets, the $\mathrm{X}: \mathrm{H}_{2}$ molar ratio can vary by several orders of magnitude as a function of the total mass of planets, i.e., the mass of gas $\left(\mathrm{H}_{2}+\mathrm{He}\right)$, the radiation, composition of planetesimals, and the assumption on the formation of clathrates and refractory organics in the disks. As can be seen in the figures, the fraction of gas $\left(\mathrm{H}_{2}\right.$ and $\left.\mathrm{He}\right)$ increases (the mass ratio of solids $M_{p l}^{s}$ relative to the mass of planets $M_{p l}^{\text {all }}$ decreases) with the total mass of planets, a result typical of planets formed in the core-accretion model. The ratio decreases slightly with the increase of the total mass of planets, leading to ISM values of abundances of species for high masses of planets.

Our results are in good agreement with abundances of observed species C, N, and S for Jupiter and Saturn, even if our data are mostly above the observational data of the two planets. Our data were obtained assuming that all volatile species are in the gas phase and homogeneously distributed in the atmosphere of planets, leading to overestimation of the abundance that could be observed in Jovian planets and future extrasolar planets. The abundance of atoms observed in the atmospheres of Jovian planets could not represent the overall abundance in the planet: some chemical species could exist partially as solid state deeper in the interior of planets, and partially as gas state in the atmosphere of planets as a function of the temperature, pressure, and type of volatile molecules. Moreover, global circulations in the atmosphere of planets can lead to horizontal/vertical mixing and cause departures from equilibrium chemistry (Baraffe et al. 2010), which could lead to discrepancies between abundances of species in cool and hot regions of planets. Our model also allows us to reproduce the enrichment of noble gas $\mathrm{Xe}, \mathrm{Kr}$, and $\mathrm{Ar}$ in Jupiter.

Abundances of atoms $\mathrm{O}$ and $\mathrm{C}$, and $\mathrm{C}: \mathrm{O}$ ratio in volatile species. Figures 12 and 13 present the average ratio of the $\mathrm{O}$ (called $\mathrm{O}^{\text {ices}}$ ) and $\mathrm{C}$ (called $\mathrm{C}^{\text {ices }}$ ) atoms in volatile species relative to all atoms $\mathrm{O}\left(\right.$ called $\left.\mathrm{O}^{\text {all }}\right)$ and $\mathrm{C}\left(\right.$ called $\left.\mathrm{C}^{\text {all }}\right)$ respectively in planets, for non-irradiated and irradiated models, and with and without (only in Fig. 12 for atoms O) refractory organics, taking into account all chemical variations $\left(\mathrm{CO}: \mathrm{CO}_{2}\right.$ variation, and clathrate formation). For non-irradiated models, volatile species count for at least $46.3 \pm 10 \%$ (resp. $66 \pm 13 \%$ ) of total atoms $\mathrm{O}$, including minerals, organics, and volatile molecules in icy/ocean planets with (resp. without) refractory organics. They also include at least $29 \pm 10 \%$ (resp. $100 \pm 6 \%$ ) of total atoms C for models with (resp. without) refractory organics. For giant planets, the average abundance of atoms is slighly lower by about $5-7 \%$ with $41.5 \pm 14 \%$ (resp. $60 \pm 18 \%$ ) for oxygen, and $22.7 \pm 12 \%$ (resp. $100 \pm 12 \%$ ) for carbon in models with (resp. without) refractory organics. Planets without refractory organics show higher abundances of $\mathrm{O}$ and $\mathrm{C}$ in ices. This means that observations of volatile species in planets poor in refractory organics show abundances of atoms in good agreement with that of the full planet. For irradiated models, the abundances of atoms $\mathrm{O}$ and $\mathrm{C}$ show lower values with radial variations (see Table 2 and Fig. 12).

One of the most important atomic ratios for the chemistry and mineralogy (see Paper I) of planets is the $\mathrm{C} / \mathrm{O}$ ratio since it controls the distribution of carbon species. To characterize the depletion or enrichment of the $\mathrm{C}: \mathrm{O}$ ratio in planets compared to the star, in Fig. 14 we plot the $\mathrm{C}: \mathrm{O}$ ratio in the atmosphere (the main observable that we have on these objects) of planets (considering only ices, and assuming that all ices are in the gas phase) and the total $\mathrm{C}: \mathrm{O}$ ratio of planets (considering ices, minerals, and refractory organics). Figure 14 presents the average $\mathrm{C}: \mathrm{O}$ molar ratio in volatile molecules (right scale) and its deviation relative to the ISM value ${ }^{3}$ (molar ratio $\frac{(\mathrm{C} / \mathrm{O})^{\text {ices }}-0.5}{0.5}$, left scale) as a function of the final semi-major axis $a_{p l}$ and the

3 The C:O molar ratio in the early stellar system (solar system composition) is 0.5 (Lodders 2003). 

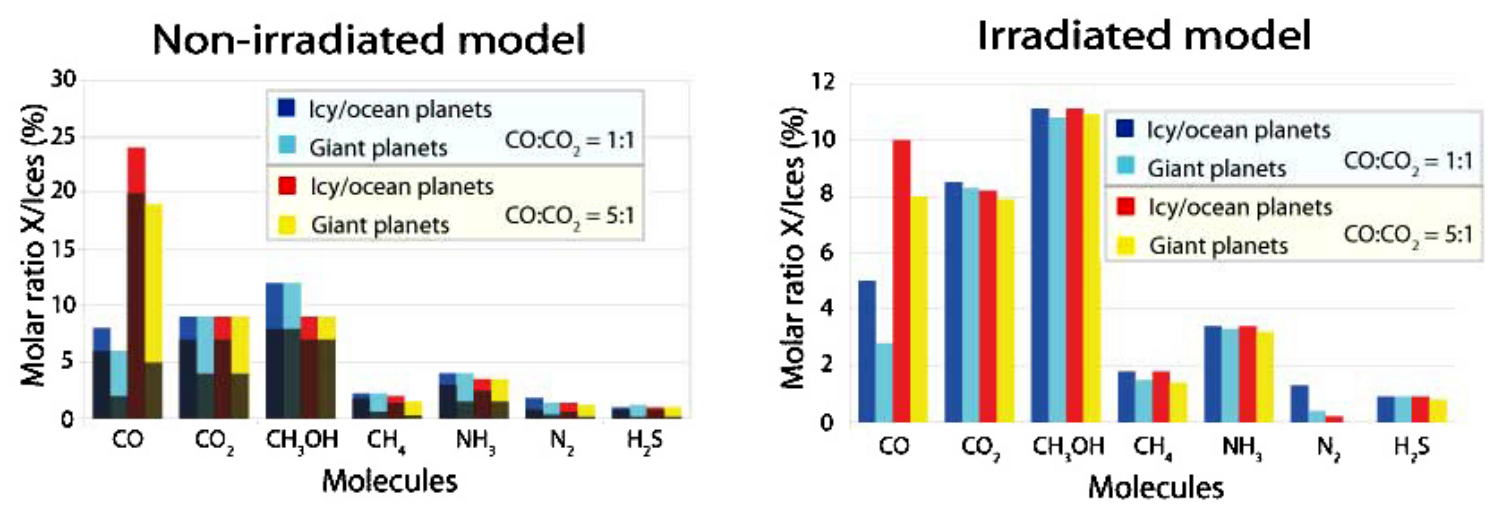

Fig. 10. Minimum (dark area) and maximum (light area) molar ratios of species $X$ relative to ices (the sum of $\mathrm{H}_{2} \mathrm{O}, \mathrm{CO}, \mathrm{CO}_{2}, \mathrm{CH}_{3} \mathrm{OH}, \mathrm{CH}_{4}, \mathrm{NH}_{3}$, $\mathrm{N}_{2}$, and $\mathrm{H}_{2} \mathrm{~S}$ ) in icy/ocean planets and giant planets for all models.

Table 2. Average molar abundance (with standard deviation) of atoms $X$ in planets.

\begin{tabular}{|c|c|c|c|c|c|c|c|c|}
\hline \multirow{3}{*}{$\begin{array}{l}\text { Models } \\
\text { planets } \\
\text { Ref. organics }\end{array}$} & \multicolumn{4}{|c|}{ Non-irradiated } & \multicolumn{4}{|c|}{ Irradiated } \\
\hline & \multicolumn{2}{|c|}{ Icy/Ocean } & \multicolumn{2}{|c|}{ Giant } & \multicolumn{2}{|c|}{ Icy/Ocean } & \multicolumn{2}{|c|}{ Giant } \\
\hline & With & Without & With & Without & With & Without & With & Without \\
\hline $\mathrm{O}^{\text {ices }} / \mathrm{O}^{\text {all } *}(\%)$ & $46.3 \pm 10$ & $66 \pm 13$ & $41.5 \pm 14$ & $60 \pm 18$ & $38.5 \pm 12.7$ & $59 \pm 14$ & $29 \pm 20$ & $45 \pm 30$ \\
\hline $\mathrm{C}^{\text {ices }} / \mathrm{C}^{\text {all* }}(\%)$ & $29 \pm 10$ & $100 \pm 6$ & $22.7 \pm 12$ & $100 \pm 12$ & $14.4 \pm 11.6$ & $98 \pm 26$ & $7 \pm 9$ & $95 \pm 42$ \\
\hline $\mathrm{C} / \mathrm{O}^{* *}$ (ices) & \multicolumn{2}{|c|}{$0.31 \pm 0.14$} & \multicolumn{2}{|c|}{$0.27 \pm 0.12$} & \multicolumn{2}{|c|}{$0.17 \pm 0.11$} & \multicolumn{2}{|c|}{$0.10 \pm 0.09$} \\
\hline $\mathrm{C} / \mathrm{O}^{* *}$ (all) & $0.49 \pm 0.08$ & $0.21 \pm 0.09$ & $\approx 0.50 \pm 0.11$ & $0.16 \pm 0.085$ & $0.47 \pm 0.054$ & $0.10 \pm 0.08$ & $0.50 \pm 0.12$ & $0.051 \pm 0.06$ \\
\hline
\end{tabular}

Notes. ${ }^{(*)}$ Average molar ratio of atoms $\mathrm{X}$ in ices relative to total atoms $X$ in planets $\left(\mathrm{X}_{p}^{\mathrm{ices}} / \mathrm{X}_{p}^{\text {all }}\right) .{ }^{(* *)}$ The molar stellar value of $\mathrm{C} / \mathrm{O}$ in our study is 0.5 .

mass of solids $M_{p l}^{s}$ in planets, for non-irradiated (upper panel) and irradiated (lower panel) models, taking into account all chemical variations ( $\mathrm{CO}: \mathrm{CO}_{2}$ variation and clathrate formation). The C:O molar ratio in volatile molecules reaches $0.31 \pm 0.14$ for icy/ocean planets with a deviation of $-40 \mp 30 \%$ relative to the ISM value ${ }^{3}$ in non-irradiated models. For giant planets, the $\mathrm{C}: \mathrm{O}$ ratio is slightly lower $(0.27 \pm 0.12)$ with a deviation of $-40 \mp 20 \%$. In irradiated models, all planets present lower values of the $\mathrm{C}: \mathrm{O}$ molar ratio (values divided by about 2 compared to non-irradiated models), and with radial variations from 0 near the star to $0.17 \pm 0.11$ (deviation of $-60 \mp 10 \%$ ) far. This means that the $\mathrm{C}: \mathrm{O}$ ratio in volatile species that could be observed in the atmosphere of planets (and assuming that all volatile species are in the gas phase) range from deviations of $-40 \pm 30 \%$ to $0 \%$ (the stellar value ${ }^{3}$ ).

Figure 15 presents the average $\mathrm{C}: \mathrm{O}$ molar ratio in all elements (ices, minerals, refractory organics) of planets (right scale) and its deviation (molar ratio $\frac{(\mathrm{C} / \mathrm{O})^{\text {all }}-0.5}{0.5}$, left scale) relative to the ISM value ${ }^{3}$ as a function of the final semi-major axis $a_{p l}$ and the mass of solids $M_{p l}^{s}$ in planets, for non-irradiated (left panel) and irradiated (right panel) models, with (upper panel) and without (lower panel) refractory organics, and taking into account all chemical variations $\left(\mathrm{CO}: \mathrm{CO}_{2}\right.$ variation and clathrate formation). Icy/ocean and giant planets with refractory organics show C:O molar ratio roughly equal to $0.5 \pm 0.1$ with a deviation of $\pm 10 \%$ relative to the stellar value. Planets without refractory organics in non-irradiated models present lower $\mathrm{C}: \mathrm{O}$ values at $0.21 \pm 0.09$ (deviation of $-60 \mp 20 \%$ ) for icy/ocean planets and $0.16 \pm 0.085$ (deviation of $-70 \mp 20 \%$ ) for giants. Planets in irradiated disks present values of the $\mathrm{C} / \mathrm{O}$ that are approximately 2 and 3 times lower for icy/ocean and giant planets, respectively.
If planets contain a large total amount of refractory organics, the C:O ratio should be of the same order of magnitude as the stellar value. Otherwise, the value should decrease by several tens of percent compared to the stellar value. We note that several rocky planets with refractory organics show high values of the $\mathrm{C}: \mathrm{O}$ ratio, up to 3 . This value corresponds to the $\mathrm{C}: \mathrm{O}$ ratio in refractory organics. Without these refractory organics, the ratio decreases to 0 in rocky planets. Table 2 summarizes the average abundances (with standard deviation) of atoms $\mathrm{O}, \mathrm{C}$, and molar ratio $\mathrm{C} / \mathrm{O}$ (in ices and all components) for all models, taking into account all chemical variations (formation of clathrates and $\mathrm{CO}: \mathrm{CO}_{2}$ variation).

\section{Types of planets formed and the boundary for the models used}

We now discuss the type of planets obtained, their possible physical evolution, and assumptions made in this study.

\subsection{Earth-like to super-Earth planets}

Planets with mass of solid components $\mathrm{M}_{p l}^{s}$ varying from $0.01 M_{\oplus}$ (dwarf planets) to about $10 M_{\oplus}$ (usually designated super-Earth) are not massive enough to accrete a large gaseous envelope onto the central core $\left(\mathrm{H}_{2}+\mathrm{He}\right)$. These planet do not have a primitive gaseous atmosphere $\left(\mathrm{H}_{2}+\mathrm{He}\right)$ or the gaseous envelope of $\mathrm{H}_{2}+\mathrm{He}$ accreted by these planets corresponds to less than $10 \%$ of the mass of solid components $\mathrm{M}_{p l}^{s}$.

Earth-like planets. The rocky planets formed near stars $\left(a_{p l} \leq\right.$ 0.4-2 AU) are mainly dry objects. However, these objects are not completely water-free. For the Earth, $\mathrm{H}_{2} \mathrm{O}$ represents 

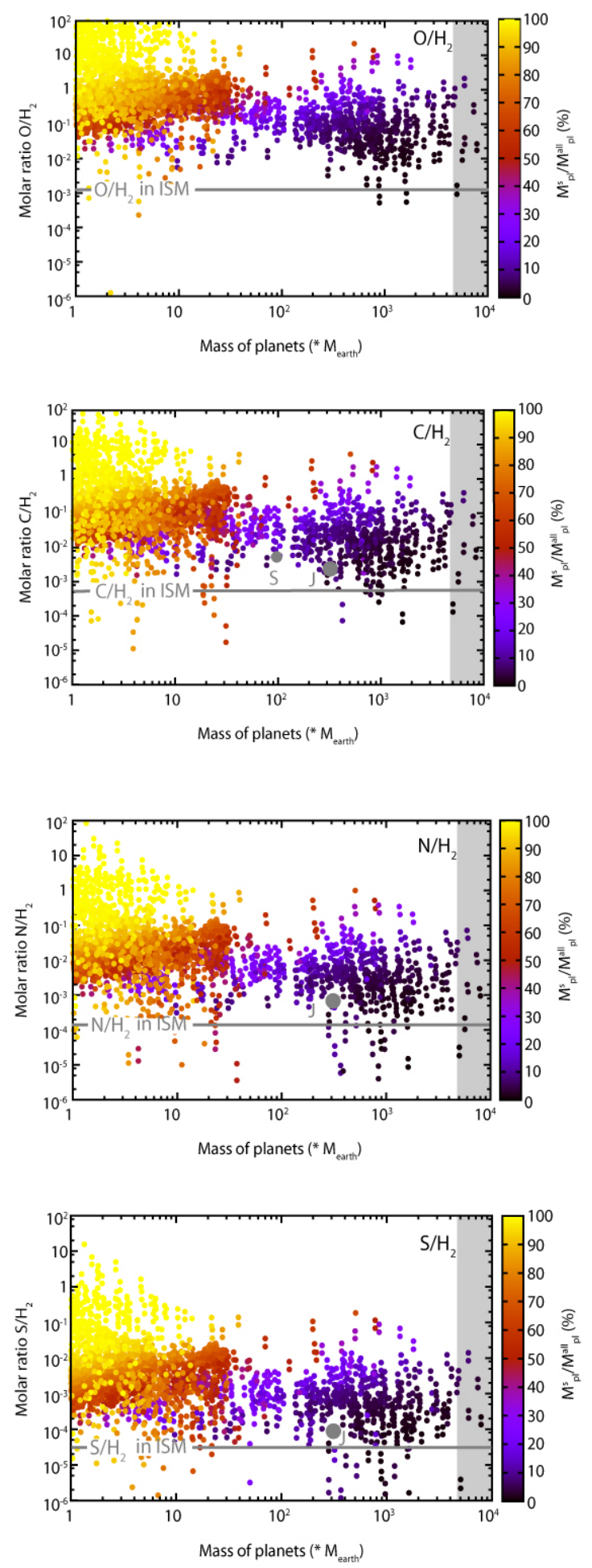

Fig. 11. Molar ratio of atoms $\mathrm{O}, \mathrm{C}, \mathrm{N}$, and $\mathrm{S}$ relative to the total mass of gas $\mathrm{H}_{2}$ in planets as a function of the total mass $M_{p l}^{\text {all }}$ of planets and mass ratio of solids $M_{p l}^{s}$ relative to total planetary mass $M_{p l}^{\text {all }}$, for all models. Horizontal gray lines represent abundances of atoms adopted in the ISM. Gray dots $J$ and $S$ represent, respectively, the abundances for Jupiter and Saturn. Data for Jupiter and Saturn provided by Wong et al. (2004), Flasar et al. (2005), Orton et al. (2005), and Briggs \& Sackett (1989). The light gray area on the right corresponds to the zone where the mass of planets is higher than 13 Jovian masses and where there could be more brown dwarfs than giant planets.

about $0.02 \%$ of the core (Bell 2010). Despite its small relative abundance, water has dramatic effects on our planet's geology, geochemistry, climate, and biology and has important implications for planetary evolution and appearance of life (Bell 2010). Most rocky planets formed in the simulation have final semimajor axes below 0.4-2 AU, but there are some rocky planets beyond 2 AU. After migration, some rocky planets are within the habitable zone, typically in the range $95-135 \%$ of the midline, based mostly on analogy with our own solar system (Bell 2010). Post-formation degassing, comet accretion, or oxidization processes can only produce a tenuous gaseous atmosphere, with no significant consequences for the planet's contraction (Baraffe et al. 2010).

Icy/ocean planets. Icy/ocean planets with mass of solid components $M_{p l}^{s}$ below about 5-10 $M_{\oplus}$ are located beyond 0.04-2 AU. Some icy/ocean planets are within the inner and outer boundaries part of the habitable zone. These planets are composed of at least $23 \pm 10 \mathrm{wt} \%$ of volatile molecules such as $\mathrm{H}_{2} \mathrm{O}, \mathrm{CO}_{2}, \mathrm{CO}, \mathrm{CH}_{3} \mathrm{OH}$, and $\mathrm{NH}_{3}$, and have an ice/rock mass ratio varying from about $0.3 \pm 0.16$ to $1.01 \pm 0.33$. Such values match those of some icy satelites and KBOs in the solar system: densities of Jovian and Saturn icy moons Ganymede, Callisto, and Titan, and Kuiper Belt objects Triton, Eris, and the PlutoCharon binary $\left(\approx 1.8-2.3 \mathrm{~g} \mathrm{~cm}^{-3}\right)$ suggest that rock and ices have comparable masses (Showman \& Malhotra 1999; Buie et al. 2006; Person et al. 2006; Brown \& Schaller 2007; McKinnon et al. 2008; Lunine et al. 2010; Sotin et al. 2010). Far away from the star where the temperature is low, planets are mainly icy with probably a tenuous atmosphere of highly volatile molecules such as $\mathrm{CO}$ and/or $\mathrm{N}_{2}$ such as dwarf planets Charon and Pluto in the solar system or liquid or frozen planets such as Europa (one of Jupiter's moons) where tidal heating from Jupiter provides the energy source that would prevent a subsurface ocean from freezing solid (Bell 2010).

Near the star, where the temperature can be higher following the luminosity of the star, only the post-formation sublimation or liquefaction of ices due to temperatures of planetary surfaces can produce a gaseous atmosphere whose composition should be a function of the temperature of sublimation of volatile molecules and their escaping velocity in the planetary atmosphere. Such ocean planets present a large variation of abundances of volatile species probably due to the different origins of their formation and the pathways taken in the disk during their migration: $\mathrm{H}_{2} \mathrm{O}$ can be the major volatile component or planets can include all volatile species in the same proportions as icy planets located far away from the star. We also note that warm temperatures could lead to chemical changes modifying the composition of the atmosphere. Such close-in and volatile rich super-Earths have already been observed such as GJ $1214 \mathrm{~b}\left(6.55 \pm 0.98 M_{\oplus}\right.$, Miller-Ricci Kempton et al. 2012) and HD 97658b (8.2 $\pm 1.2 M_{\oplus}$, Dragomir et al. 2013). Note, however, that these planets orbit stars with lower masses and lower luminosities. Rogers \& Seager (2010) found that GJ 1214b, a planet orbiting a star of mass equal to $0.157 M_{\odot}$ (Charbonneau et al. 2009) and a luminosity equal to $0.3 \%$ the Sun's, must be a water-rich planet composed of at least $60 \%$ water by mass with a thick steam atmosphere to reproduce the planet's observed radius $\left(2.68 \pm 0.13 R_{\oplus}\right.$, Charbonneau et al. 2009). However, an atmosphere composed of a combination of water vapor and hydrogen gas is also consistent with the planet's observed mass and radius, and these would require a smaller fraction of water to reproduce the planet's bulk density (Kempton et al. 2012). By examining all possible mixtures of water and $\mathrm{H} / \mathrm{He}$, Valencia et al. (2013) found that the bulk amount of $\mathrm{H} / \mathrm{He}$ in GJ $1214 \mathrm{~b}$ is at most $7 \%$ by mass. 

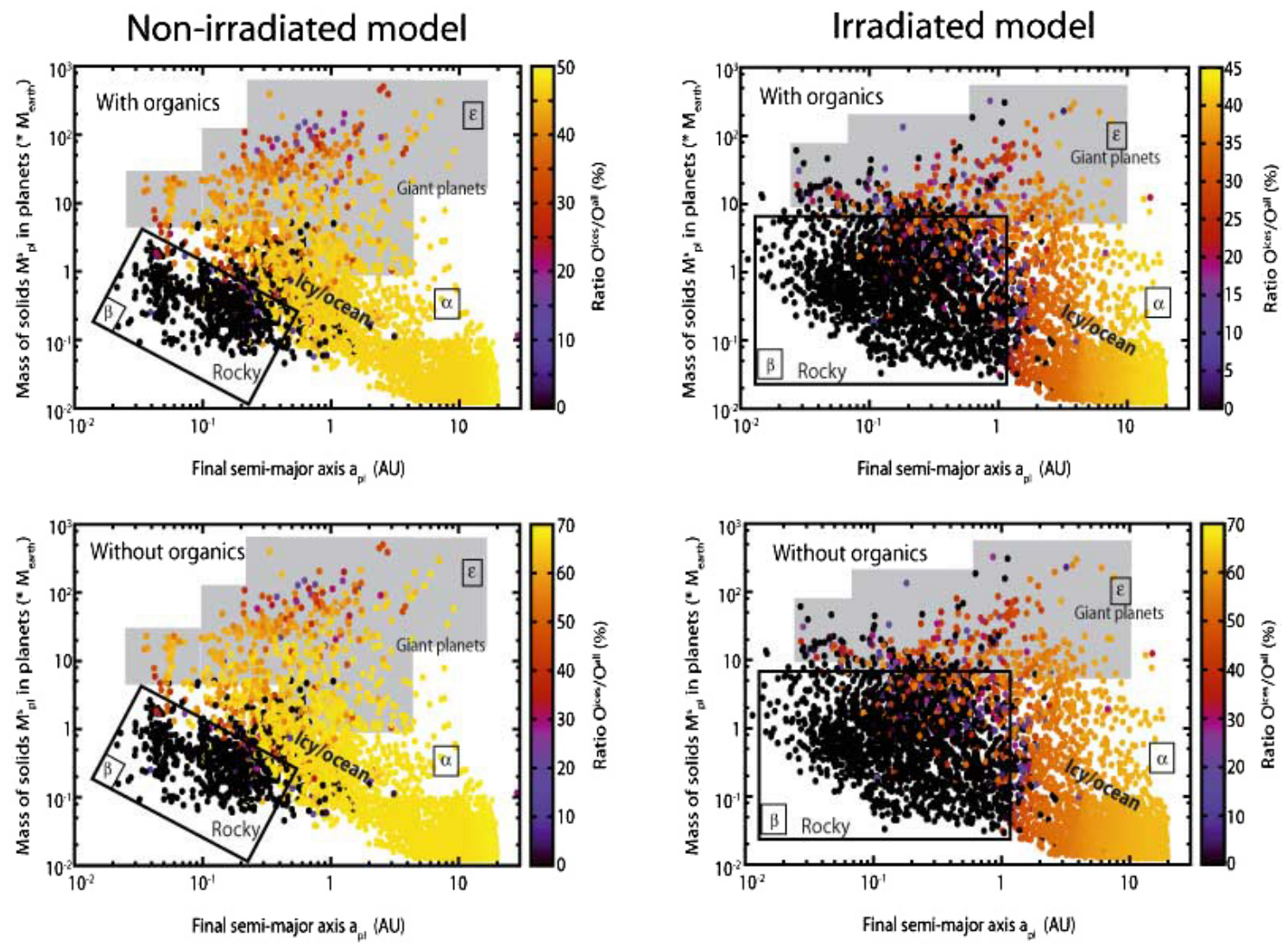

Fig. 12. Average ratio of atoms $\mathrm{O}^{\mathrm{ices}}$ in volatile molecules of planets relative to atoms $\mathrm{O}^{\text {all }}$ in all solid components as a function of the solid mass $M_{p l}^{s}$ of planets and final semi-major axis $a_{p l}$, for non-irradiated (left panel) and irradiated (right panel) models, both with (upper panel) and without (lower panel) refractory organics, taking into account all chemical variations ( $\mathrm{CO}: \mathrm{CO}_{2}$ variation and clathrate formation).

Interestingly, our models, applied to sun mass and sun luminosity, show that some planets below 0.1 AU can have water mass ratio ranging from $10 \%$ to $50 \%$. Applying our models to stars of lower mass and lower luminosity with different abundances of atoms could match the composition and position of planets observed more accurately.

\subsection{Super-Earth to giant planets}

Planets with mass of solid components $M_{p l}^{s}$ higher than 5-10 $M_{\oplus}$ (usually denominated as super-Earth) which are massive enough to accrete a large gaseous envelope onto the central core $\left(\mathrm{H}_{2}+\right.$ $\mathrm{He}$ ) are labeled Neptune-like or gas giant planets. The gaseous envelope of $\mathrm{H}_{2}+\mathrm{He}$ accreted by these planets correspond to values much higher than $10 \%$ of the mass of solid components $M_{p l}^{s}$.

Super-Earth planets to Neptune-like planets. Planets with mass of solid components $\mathrm{M}_{p l}^{s}$ varying from $5 M_{\oplus}$ to about $20 M_{\oplus}$ usually denominated as super-Earth down to Earthlike planets, are not massive enough to lead to rapid accretion (runaway regime) of a large gaseous envelope onto the central core. However, terrestrial planets can accrete a gaseous envelope of $\mathrm{H}_{2}+\mathrm{He}$ corresponding to up to $100 \mathrm{wt} \%$ of the mass of solid components $M_{p l}^{s}$, leading to planets composed of $50 \mathrm{wt} \%$ of solids (ices, minerals, refractory organics) and $50 \mathrm{wt} \%$ of gas $\mathrm{H}_{2}+\mathrm{He}$. This leads to formation of Neptune- and Uranus-like planets. For these planets, the gaseous envelope essentially governs the gravothermal evolution of the planet (Bell 2010). However, differentiation, outgassing, and additional late heavy bombardment of icy planetesimals and subsequent accretion of chemical species could result in the enrichment of volatile molecules in their atmospheres over time, while $\mathrm{H}_{2}$ could slowly be lost because of escape velocity and solar wind breakdown. This could then lead over time to the enrichment of the abundances of volatile molecules (relative to $\mathrm{H}_{2}$ ) in the gaseous atmosphere of these planets. The abundance of species, i.e., the chemical reactions, in the gas envelope (dominated by $\mathrm{H}_{2}$ and $\mathrm{He}$ ) of these planets is temperature-pressure dependant and could change significantly with close-in planets $(\leq 0.1 \mathrm{AU})$ which could be affected by high temperatures. Closein Neptune-like planets, produced in our model, and called as hot Neptunes, have already been observed, such as the planets GJ 436b (23 $M_{\oplus}$, Agúndez et al. 2014) and HAT-P-11b (23 $M_{\oplus}$, Lecavelier des Etangs et al. 2013). Note however that these planets orbit stars with significantly lower masses and luminosities, and different atom abundances. GJ 436b, a planet orbiting a star of mass equal to $0.41 M_{\odot}$ and a luminosity equal to $2.5 \%$ the Sun's (Butler et al. 2004), at about 0.03 AU, has a planetary effective temperature of about 700-800 K (Deming et al. 2007; Demory et al. 2007; Stevenson et al. 2010) and an atmosphere rich in $\mathrm{CO}$, but poor in $\mathrm{CH}_{4}$ and $\mathrm{H}_{2} \mathrm{O}$ (Stevenson et al. 2010; Madhusudhan \& Seager 2011; and Moses et al. 2013). Our model does not predict such a composition of planets, but chemical processes at high temperature ${ }^{4}$ that change the abundances of volatile molecules are not taken into account in this study since we produce only the original bulk composition derived from planetesimals.

\footnotetext{
4 Such as described by Eqs. (1)-(3) in the atmosphere of gas giant planets, see Paper II.
} 

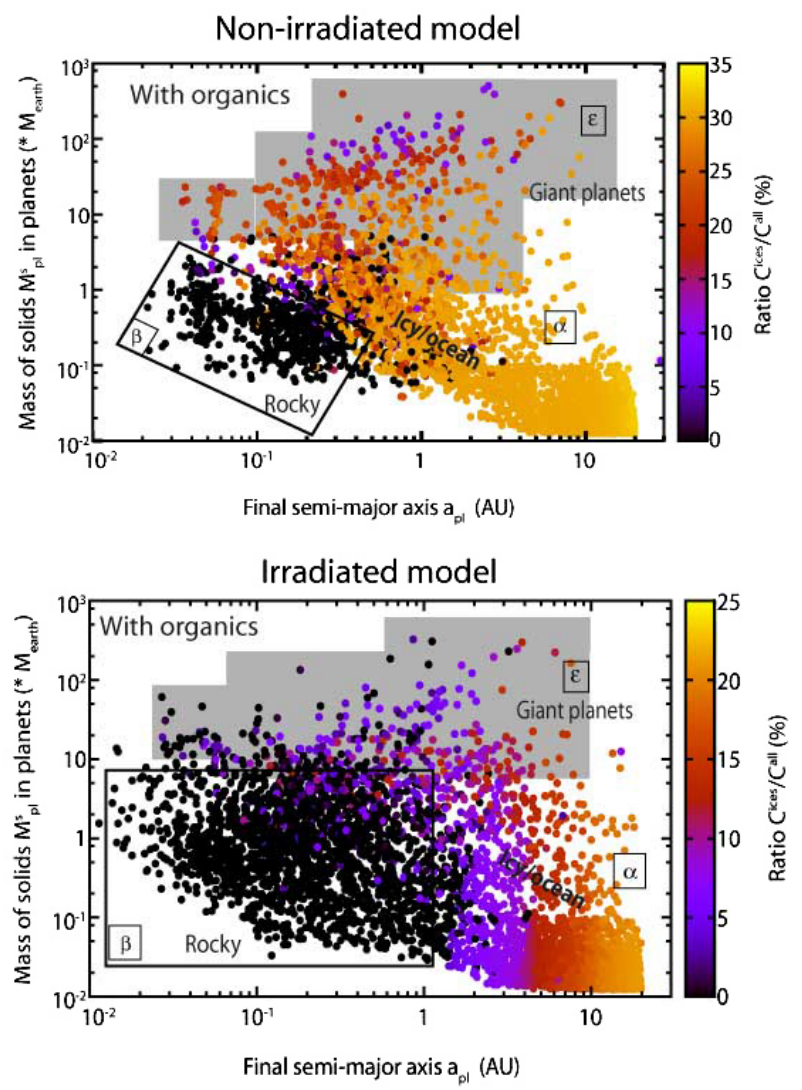

Fig. 13. Same as in Fig. 12 but for $\mathrm{C}$ atoms and with refractory organics only.

Neptune-like to super-Jupiter planets. Gas giant planets with mass of solids varying from $10-20 M_{\oplus}$ to $500 M_{\oplus}$ have a primitive gaseous atmosphere $\left(\mathrm{H}_{2}+\mathrm{He}\right)$, with mass ratio of solid components $M_{p l}^{s}$ relative to the total mass $M_{p l}^{\text {all }}$ of planets ranging from $60 \mathrm{wt} \%$ to values lower than $10 \mathrm{wt} \%$. This leads to planets with ice/rock mass ratios varying from roughly 0.1 to 1.5 . Our results for the ice/rock mass ratio in gas giant planets are slightly lower than those assumed in theoretical studies of giant planet formation as explained in Paper II. When gas water molecules condense, the solid surface density $\Sigma$ of the disk is assumed to increase by a factor of 3-4 (Lecar et al. 2006; Encrenaz 2008). This assumption helps gas giant planets to form rapidly before the quick dissipation of the gas in the disk on a timescale of a few million years due to accretion onto the central protostar and evaporation from the protostar's irradiation. However, our results show that the ice/rock mass ratio in planetesimals (Paper II) and planets is on the order of magnitude $1 \pm 0.5$, following the different assumptions on the thermodynamic conditions of formation of ices in the disks (see Paper II), leading to an increase in only the solid components by approximately $1.5-2.5$ beyond the ice line, which is a factor lower by $2 \pm 0.5$ than our best value compared to the one indicated by papers of planet formation (Hayashi 1981; Stevenson \& Lunine 1988; Encrenaz 2008). This discrepancy with our results can be explained mainly by the use by previous models (Hayashi 1981) of solar atomic abundances of Cameron (1970) that are higher by 50-90\% for atoms C, O, and N compared to Lodders's (2003) abundances taken into account in this study. Moreover, Hayashi (1981) made some assumptions on the volatile species, taking into account only $\mathrm{H}_{2} \mathrm{O}$, $\mathrm{CH}_{4}$, and $\mathrm{NH}_{3}$, all condensing together at $170 \mathrm{~K}$ (see Paper II).
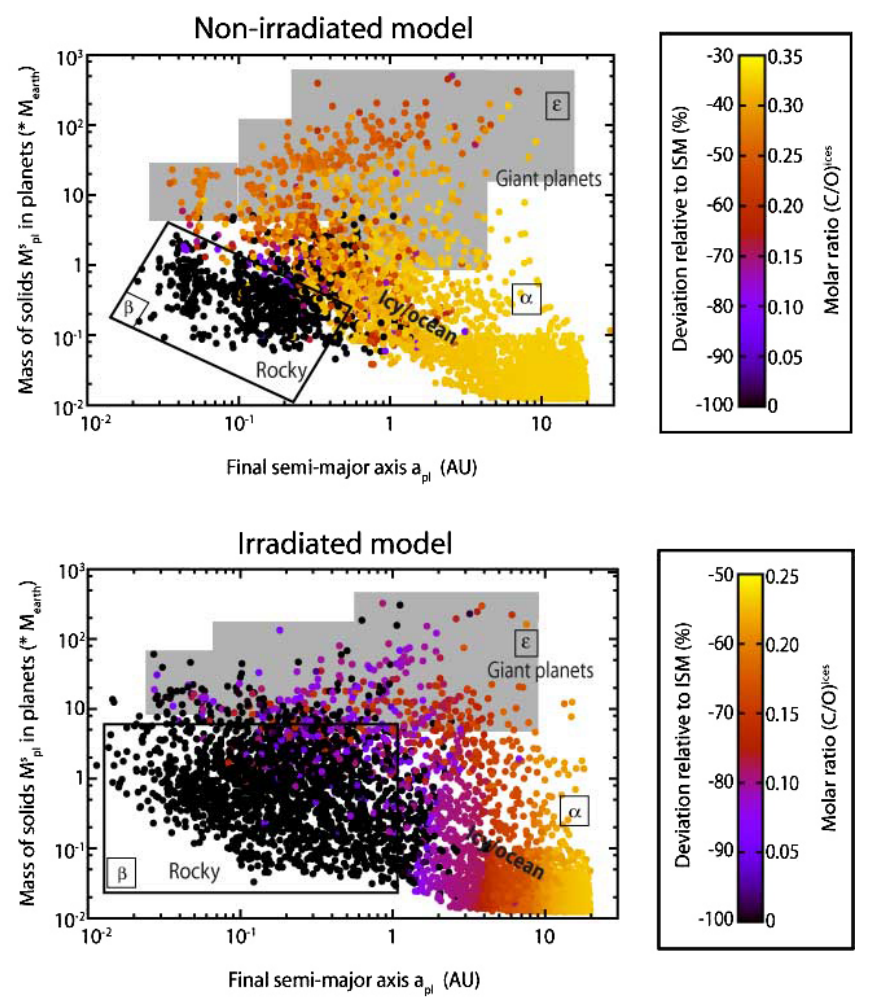

Fig. 14. Average molar ratio $\mathrm{C}: \mathrm{O}$ in volatile species as a function of the solid mass $M_{p l}^{s}$ of planets and final semi-major axis $a_{p l}$. Non-irradiated (upper panel) and irradiated (lower panel) models taking into account all chemical variations ( $\mathrm{CO}: \mathrm{CO}_{2}$ variation and clathrate formation).

Theoretical models of Jupiter's interior predict that the total mass of heavy elements ranges from $20 M_{\oplus}$ to $40 M_{\oplus}$ (Saumon \& Guillot 2004; Nettelmann et al. 2008; Militzer et al. 2008; Helled \& Schubert 2009), leading to $M_{p l}^{s}: M_{p l}^{\text {all }}$ mass ratio ranging from $6 \%$ to $13 \%$ for this planet, in good agreement with results of the model of Alibert et al. (2013). However, our results on the ice/rock mass ratio are slightly different from those of the internal structure models of Uranus and Neptune (Podolak et al. 1995). Three-layer models with a central rocky core, an ice layer, and an outer $\mathrm{H} / \mathrm{He}$ envelope suggest an overall composition of $25 \mathrm{wt} \%$ of rocks, $60-70 \mathrm{wt} \%$ of ices, and 5-15 wt\% of gaseous H/He (Podolak et al. 1991; Baraffe et al. 2010). Other solutions exist, as suggested by Podolak et al. (1995), assuming not a pure ice second layer, but a mixture of ices, rock (minerals + refractory organics), and gas. However, the ice/rock mass ratio remains at about 2.5 for both planets Uranus and Neptune in these models, $\approx 1.5$ times higher that our best results. In the solar system, the largest reservoirs of water should be Jupiter and Saturn which represent $91 \%$ of the planetary mass in our solar system (Fortney \& Nettelmann 2010), and Uranus and Neptune (7\% of the planetary mass in our solar system). However, there are no data available on the abundance of tropospheric or deeper ices (mainly $\mathrm{H}_{2} \mathrm{O}$ ) on these planets. The bulk compositions of these planets are not well constrained (Podolak et al. 1995; Marley et al. 1995; Helled et al. 2011; Podolak \& Helled 2012). In fact, it is still unclear what the mass fraction of ices is in Uranus and Neptune, despite their categorization as icy planets (Podolak \& Helled 2012).

Measurements by the Galileo probe mass spectrometer suggest that the atmosphere of Jupiter is enriched in heavy elements by a factor of up to 3 compared with the Sun (Young 2003; Helled \& Schubert 2009). Our results suggest that the 

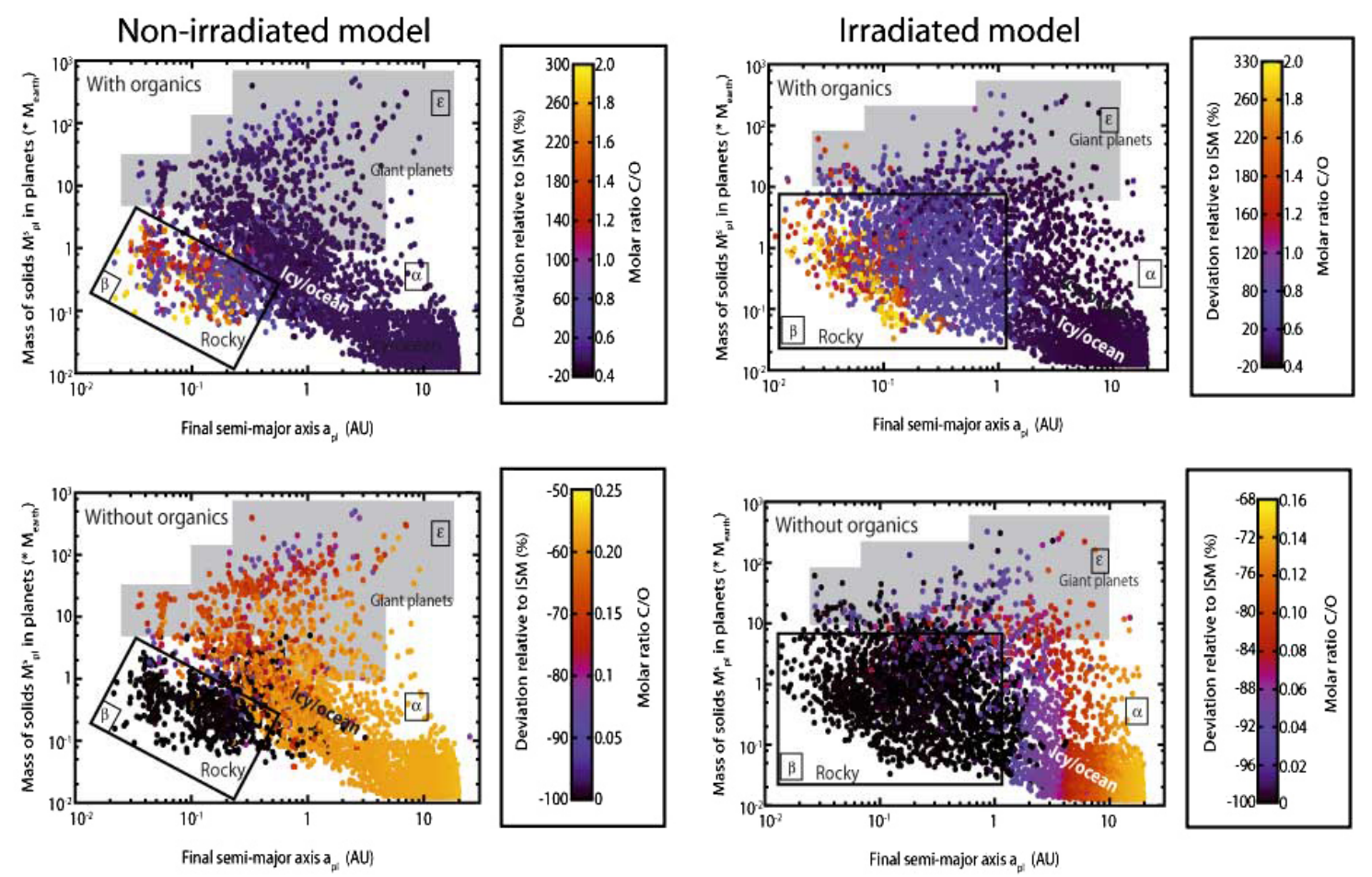

Fig. 15. Same as Fig. 14 but for the average molar ratio $\mathrm{C}: \mathrm{O}$ in all solid components of planets.

abundances of atoms (relative to $\mathrm{H}_{2}$ ) in giant planets are almost higher that the stellar values (up to 3 orders of magnitude for low mass planets), but decrease with an increase in the total mass of planets, leading to stellar values for large planet masses which means there could be more brown dwarfs than giant planets for total masses higher than 13 Jupiter masses. The abundances of species (relative to $\mathrm{H}_{2}$ ) in the atmosphere of giant planets determined in this work are upper limits. First, we assumed that all volatile species incorporated as ices in planets were in the gaseous state of the atmosphere of planets although a part could be in the solid state, deeper in the planets, as a function of the temperature, pressure, and type of volatile molecules. Second, the heating of the atmosphere of planets (after migration toward the star) near the star could trigger slow chemical reactions such as described in Eqs. (1)-(3) in Paper II, leading to the enrichment or depletion of species in the atmosphere of planets. Moreover, global circulations of the atmosphere of planets can lead to discrepancies of abundances of species between cool and hot regions of the atmosphere.

The model of planet formation used in this work (Alibert et al. 2013) assumes small planetesimals that keep the same radius (about $100 \mathrm{~m}$ ). As shown in Fortier et al. (2013), the formation of giant planets is favored by the accretion of small planetesimals which do not evolve with time and leads to the formation of massive planets with masses higher than $500 M_{\oplus}$. Increasing the radius of planetesimals reduces severely the accretion rate and the growth of planets (see Alibert et al. 2013; Fortier et al. 2013). Assuming a size distribution for planetesimals and a radius evolution of planetesimals (increase due to accretion of smaller objects, and decrease due to the sublimation of ices when shifted toward the star) could change the mass of planets formed and presented in this study. Such considerations will be the subject of future works.

It is important to remember that the chemical compositions of planets given in this study do not take into account loss of material due to collisional heating and breaking of bodies; size of bodies and temperature of sublimation of volatile molecules (evaporation process, escape velocity of volatile molecules); tidal and radioactive heating and geysers as observed in the satellites Enceladus and Europa; and chemical processes ${ }^{5}$ changing the abundances of volatile molecules. In addition, this study does not consider the radial migration of grains and small planetesimals $(\leq 1 \mathrm{~km})$ in the disk due to the effect of gas drag and gravitational torques. We computed the chemical composition of ices in planetesimals and planets by using the thermodynamic conditions derived from an $\alpha$ disk both with and without irradiation, and for solar conditions (mass, luminosity, and composition of atoms). The irradiated and non-irradiated models used in this study do not properly model the physics in the disks. However, they allow us to frame the thermodynamic conditions of ice formation in the cooling disk and migration of planets, respectively, near and far away from the star. So, the abundance of volatile molecules of planets calculated in this work constitute an upper (resp. lower) limit for non-irradiated (resp. irradiated) models in solar conditions. The gas phase incorporated by giant planets and assumed to be composed only of $\mathrm{H}_{2}$ and $\mathrm{He}$, should be composed of other volatile species as suggested by Oberg et al. (2011). Calculating the gas composition of volatile molecules incorporated in giant planets could change slightly the chemical gas composition of these planets supposed to be derived directly from ice species in this study (see Fig. 11) and change their carbon to oxygen ratio. We currently extend our work in this direction. Finally, the chemical composition of planets and icy satellites are derived from average values of abundances of species observed in the ISM. Abundances of species can change up to several tens of percentage points for some molecules such as $\mathrm{CO}_{2}, \mathrm{CH}_{3} \mathrm{OH}$, and $\mathrm{NH}_{3}$ (see Paper II). A system with lower extremes or larger abundances of species compared to average values taken in this study could change the chemical composition of planets given in this paper.

\footnotetext{
5 Such as described by Eqs. (1)-(3) in the atmosphere of gas giant planets, see Paper II.
} 
In order to better constrain the chemical composition of planets, future work will focus on the nonsolar composition of atoms (C/O variation), different masses and luminosities of star, and will include the radial migration of grains and planetesimals in the disk. The computation of radii of planets in a self-consistent way using these results will also be done in future work.

\section{Summary}

In this work, we have computed the composition of volatile molecules incorporated in planets after migration in the disk, using 16 different physico-chemical conditions. To do this, we proceeded in two steps. In Paper II, we used models of disk accretion and ice formation to calculate the abundance of ices incorporated in planetesimals for different initial physico-chemical conditions, varying the abundance of $\mathrm{CO}$ in planetesimals, i.e., the disk, taking into account, or not, the presence of refractory organics, clathrates, star radiation, and mass of the disk. In this paper, starting from the chemical composition calculated in the disk for different surface densities, we used the model of planet formation and migration of Alibert et al. (2013) to determine a wide variety of chemical composition of planets listed in three groups:

- Giant planets with mass of solids 5-10 $M_{\oplus} \leq M_{p l}^{s} \leq 500 M_{\oplus}$

- Icy/ocean planets with mass of solids $M_{p l}^{s} \leq 5-10 M_{\oplus}$.

- Rocky planets with mass of solids $M_{p l}^{s} \leq 5-10 M_{\oplus}$, and mainly located inside $2 \mathrm{AU}$.

The main results regarding the chemical composition of these planets are described below.

1. Icy/ocean planets do not migrate much and retain approximately the same chemical composition as planetesimals beyond the ice lines.

2. Giant planets show high variations of abundances of all volatile species and some of them are depleted in ices relative to icy/ocean planets. Many giant planets in non-irradiated models have lower abundances of highly volatile species such as $\mathrm{CH}_{4}, \mathrm{CO}$, and $\mathrm{N}_{2}$, and most of them in irradiated models are depleted in these species compared to icy/ocean planets.

3. Ice/rock and Ice/all elements ratio:

- The mass fraction of ices varies from $23 \pm 10$ to $50 \pm$ $12 \mathrm{wt} \%$ in icy/ocean planets. This value decreases to less than $0.01 \mathrm{wt} \%$ in rocky planets and can reach up to $43 \pm$ $17 \%$ in giant planets.

- The ice/rock mass ratio in planets varies from 0 (rocky planets) to 1.5 (icy/ocean and gas giant planets).

4. Chemical composition of planets:

- The dominant chemical species in ices remains $\mathrm{H}_{2} \mathrm{O}$ (at least $63 \pm 14 \%$ in mol relative to all ices) whatever the type of planets formed and assumptions on the disk, the chemical composition, and evolution.

- The three dominant molecules after $\mathrm{H}_{2} \mathrm{O}$ are carbonbearing species such as $\mathrm{CO}, \mathrm{CO}_{2}$, and $\mathrm{CH}_{3} \mathrm{OH}$.

- The dominant nitrogen-bearing species remains $\mathrm{NH}_{3}$.

5. Atomic composition of planets:

- The abundance of atoms $\mathrm{O}, \mathrm{C}, \mathrm{N}$, and $\mathrm{S}$ (relative to $\mathrm{H}_{2}$ ) in the atmosphere of gas giant planets is almost greater than the stellar values for most planets, but decreases slightly with the increase of the total mass of planets.

- Our results on the abundance of atoms C, N, and S (relative to $\mathrm{H}_{2}$ ) are in good agreement with the abundances observed for Jupiter and Saturn.
- Taking into account all physico-chemical variations, the $\mathrm{C}: \mathrm{O}$ molar ratio in volatile molecules reaches up to $0.31 \pm$ 0.14 for icy/ocean planets, and $0.27 \pm 0.12$ for gas giant planets, about $40-50 \%$ of the stellar value.

- The total C:O molar ratio in planets (considering ices, minerals, and refractory organics) reaches up to $0.5 \pm 0.1$ considering refractory organics, and $0.21 \pm 0.09$ without, about $0-60 \%$ of the stellar value.

Acknowledgements. This work has been supported by the Swiss National Science Foundation, the Center for Space and Habitability of the University of Bern and the European Research Council under grant 239605.

\section{References}

Agúndez, M., Venot, O., Selsis, F., \& Iro, N. 2014, ApJ, 781, 68

Alibert, Y., Mordasini, C., Benz, W., \& Winisdoerffer, C. 2005, A\&A, 434, 343

Alibert, Y., Carron, F., Fortier, A., et al. 2013, A\&A, 558, A109

Baraffe, I., Chabrier, G., \& Barman, T. 2010, Rep. Prog. Phys., 73, 016901

Barclay, T., Rowe, J. F., Lissauer, J. J., et al. 2013, Nature, 494, 452

Barman, T. 2007, ApJ, 661, L191

Barman, T. S. 2008, ApJ, 676, L61

Beaulieu, J. P., Carey, S., Ribas, I., \& Tinetti, G. 2008, ApJ, 677, 1343

Beaulieu, J. P., Kipping, D. M., Batista, V., et al. 2010, MNRAS, 409, 963

Bell, J. F. 2010, Highlights of Astronomy, 15, 29

Bockelée-Morvan, D., Crovisier, J., Mumma, M. J., \& Weaver, H. A. 2004, Comets II, 391

Bond, J. C., O’Brien, D. P., \& Lauretta, D. S. 2010, ApJ, 715, 1050

Brown, M. E., \& Schaller, E. L. 2007, Science, 316, 1585

Buie, M. W., Grundy, W. M., Young, E. F., Young, L. A., \& Stern, S. A. 2006, AJ, 132, 290

Burrows, A., Hubeny, I., \& Sudarsky, D. 2005, ApJ, 625, L135

Burrows, A., Hubeny, I., Budaj, J., Knutson, H. A., \& Charbonneau, D. 2007, ApJ, 668, L171

Burrows, A., Budaj, J., \& Hubeny, I. 2008, ApJ, 678, 1436

Butler, R. P., Vogt, S. S., Marcy, G. W., et al. 2004, ApJ, 617, 580

Charbonneau, D., Brown, T. M., Noyes, R. W., \& Gilliland, R. L. 2002, ApJ, 568,377

Charbonneau, D., Allen, L. E., Megeath, S. T., et al. 2005, ApJ, 626, 523

Charbonneau, D., Knutson, H. A., Barman, T., et al. 2008, ApJ, 686, 1341

Charbonneau, D., Berta, Z. K., Irwin, J., et al. 2009, Nature, 462, 891

Deming, D., Harrington, J., Laughlin, G., et al. 2007, ApJ, 667, L199

Demory, B.-O., Gillon, M., Barman, T., et al. 2007, A\&A, 475, 1125

Désert, J.-M., Lecavelier des Etangs, A., Hébrard, G., et al. 2009, ApJ, 699 478

Doty, S. D., van Dishoeck, E. F., van der Tak, F. F. S., \& Boonman, A. M. S. 2002, A\&A, 389, 446

Dragomir, D., Matthews, J. M., Eastman, J. D., et al. 2013, ApJ, 772, L2

Encrenaz, T. 2008, ARA\&A, 46, 57

Fletcher, L. N., Orton, G. S., Teanby, N. A., Irwin, P. G. J., \& Bjoraker, G. L. 2009, Icarus, 199, 351

Fortier, A., Alibert, Y., Carron, F., Benz, W., \& Dittkrist, K.-M. 2013, A\&A, 549, A44

Fortney, J. J., \& Marley, M. S. 2007, ApJ, 666, L45

Fortney, J. J., \& Nettelmann, N. 2010, Space Sci. Rev., 152, 423

Gautier, D., Hersant, F., Mousis, O., \& Lunine, J. I. 2001a, ApJ, 550, L227

Gautier, D., Hersant, F., Mousis, O., \& Lunine, J. I. 2001b, ApJ, 559, L183

Griffith, C. A., Tinetti, G., Swain, M. R., et al. 2011, EPSC-DPS Joint Meeting 2011, 140

Grillmair, C. J., Burrows, A., Charbonneau, D., et al. 2008, Nature, 456, 767

Hayashi, C. 1981, Prog. Theor. Phys. Suppl., 70, 35

Helled, R., \& Schubert, G. 2009, ApJ, 697, 1256

Helled, R., Anderson, J. D., Podolak, M., \& Schubert, G. 2011, ApJ, 726, 15

Hersant, F., Gautier, D., \& Lunine, J. I. 2004, Planet. Space Sci., 52, 623

Johnson, T. V., Mousis, O., Lunine, J. I., \& Madhusudhan, N. 2012, ApJ, 757, 192

Lecar, M., Podolak, M., Sasselov, D., \& Chiang, E. 2006, ApJ, 640, 1115

Lecavelier des Etangs, A., Sirothia, S. K., Gopal-Krishna, \& Zarka, P. 2013, A\&A, 552, A65

Lee, J.-M., Fletcher, L. N., \& Irwin, P. G. J. 2012, MNRAS, 420, 170

Lodders, K. 2003, ApJ, 591, 1220

Lunine, J. I. 2011, Mem. Soc. Astron. It., 82, 368

Lunine, J., Choukroun, M., Stevenson, D., \& Tobie, G. 2010, Titan from Cassini-

Huygens, 35 (Springer Science + Business Media)

Madhusudhan, N. 2012, ApJ, 758, 36 
Madhusudhan, N., \& Seager, S. 2009, ApJ, 707, 24

Madhusudhan, N., \& Seager, S. 2010, EGU General Assembly Conference Abstracts, 12, 14364

Madhusudhan, N., \& Seager, S. 2011, ApJ, 729, 41

Madhusudhan, N., Harrington, J., Stevenson, K. B., et al. 2011, Nature, 469, 64

Marboeuf, U., Mousis, O., Ehrenreich, D., et al. 2008, ApJ, 681, 1624

Marboeuf, U., Thiabaud, A., Alibert A., Cabral, N., \& Benz, W. 2014, A\&A, 570, A35 (Paper II)

Marley, M. S., Gómez, P., \& Podolak, M. 1995, J. Geophys. Res., 100, 23349

McKinnon, W. B., Prialnik, D., Stern, S. A., \& Coradini, A. 2008, The Solar System Beyond Neptune, 213 (Tucson, AZ: University Arizona Press)

Militzer, B., Hubbard, W. B., Vorberger, J., Tamblyn, I., \& Bonev, S. A. 2008, ApJ, 688, L45

Miller-Ricci Kempton, E., Zahnle, K., \& Fortney, J. J. 2012, ApJ, 745, 3

Mordasini, C., Alibert, Y., \& Benz, W. 2009, A\&A, 501, 1139

Mordasini, C., Alibert, Y., Klahr, H., \& Henning, T. 2012a, A\&A, 547, A111

Mordasini, C., Alibert, Y., Georgy, C., et al. 2012b, A\&A, 547, A112

Moses, J. I., Madhusudhan, N., Visscher, C., \& Freedman, R. S. 2013, ApJ, 763, 25

Mousis, O., Lunine, J. I., Picaud, S., \& Cordier, D. 2010, Faraday Discussions, 147, 509

Mumma, M. J., \& Charnley, S. B. 2011, ARA\&A, 49, 471

Nettelmann, N., Holst, B., Kietzmann, A., et al. 2008, ApJ, 683, 1217

Nomura, H., \& Millar, T. J. 2004, A\&A, 414, 409

Öberg, K. I., Murray-Clay, R., \& Bergin, E. A. 2011, ApJ, 743, L16

Person, M. J., Elliot, J. L., Gulbis, A. A. S., et al. 2006, AJ, 132, 1575

Podolak, M., \& Helled, R. 2012, ApJ, 759, L32
Podolak, M., Hubbard, W. B., \& Stevenson, D. J. 1991, Uranus, 29 (Tucson, AZ: University Arizona Press)

Podolak, M., Weizman, A., \& Marley, M. 1995, Planet. Space Sci., 43, 1517

Rogers, L. A., \& Seager, S. 2010, ApJ, 716, 1208

Saumon, D., \& Guillot, T. 2004, ApJ, 609, 1170

Showman, A. P., \& Malhotra, R. 1999, Science, 296, 77

Snellen, I. A. G., Albrecht, S., de Mooij, E. J. W., \& Le Poole, R. S. 2008, A\&A, 487,357

Sotin, C., Mitri, G., Rappaport, N., Schubert, G., \& Stevenson, D. 2010, Titan from Cassini-Huygens, 61 (Springer Science + Business Media)

Stevenson, D. J., \& Lunine, J. I. 1988, Icarus, 75, 146

Stevenson, K. B., Harrington, J., Nymeyer, S., et al. 2010, Nature, 464, 116

Swain, M. R., Vasisht, G., \& Tinetti, G. 2008, Nature, 452, 329

Swain, M. R., Tinetti, G., Vasisht, G., et al. 2009a, ApJ, 704, 1616

Swain, M. R., Vasisht, G., Tinetti, G., et al. 2009b, ApJ, 690, L114

Thiabaud, A., Marboeuf, U., Alibert, Y., et al. 2014, A\&A, 562, A27

Tinetti, G., \& Griffith, C. A. 2010, Pathways Towards Habitable Planets, ASP Conf. Ser., 430, 115

Tinetti, G., Vidal-Madjar, A., Liang, M.-C., et al. 2007, Nature, 448, 169

Tinetti, G., Beaulieu, J. P., Henning, T., et al. 2012, Exp. Astron., 34, 311

Udry, S., \& Santos, N. C. 2007, ARA\&A, 45, 397

Valencia, D., Guillot, T., Parmentier, V., \& Freedman, R. S. 2013, ApJ, 775, 10

Visser, R., Doty, S. D., \& van Dishoeck, E. F. 2011, A\&A, 534, A132

Waldmann, I. P., Tinetti, G., Drossart, P., et al. 2012, ApJ, 744, 35

Wong, M. H., Mahaffy, P. R., Atreya, S. K., Niemann, H. B., \& Owen, T. C. 2004, Icarus, 171, 153

Young, R. E. 2003, New Astron. Rev., 47, 1 